\title{
Functional cerebral blood volume mapping with simultaneous multi-slice acquisition
}

Citation for published version (APA):

Huber, L., Ivanov, D., Guidi, M., Turner, R., Uludag, K., Möller, H. E., \& Poser, B. A. (2016). Functional cerebral blood volume mapping with simultaneous multi-slice acquisition. Neuroimage, 125, 1159-1168. https://doi.org/10.1016/j.neuroimage.2015.10.082

Document status and date:

Published: 15/01/2016

DOI:

10.1016/j.neuroimage.2015.10.082

Document Version:

Accepted author manuscript (Peer reviewed / editorial board version)

Document license:

CC BY-NC-ND

\section{Please check the document version of this publication:}

- A submitted manuscript is the version of the article upon submission and before peer-review. There can be important differences between the submitted version and the official published version of record.

People interested in the research are advised to contact the author for the final version of the publication, or visit the DOI to the publisher's website.

- The final author version and the galley proof are versions of the publication after peer review.

- The final published version features the final layout of the paper including the volume, issue and page numbers.

Link to publication

\footnotetext{
General rights rights.

- You may freely distribute the URL identifying the publication in the public portal. please follow below link for the End User Agreement:

www.umlib.nl/taverne-license

Take down policy

If you believe that this document breaches copyright please contact us at:

repository@maastrichtuniversity.nl

providing details and we will investigate your claim.
}

Copyright and moral rights for the publications made accessible in the public portal are retained by the authors and/or other copyright owners and it is a condition of accessing publications that users recognise and abide by the legal requirements associated with these

- Users may download and print one copy of any publication from the public portal for the purpose of private study or research.

- You may not further distribute the material or use it for any profit-making activity or commercial gain

If the publication is distributed under the terms of Article $25 \mathrm{fa}$ of the Dutch Copyright Act, indicated by the "Taverne" license above, 


\title{
Functional Cerebral Blood Volume Mapping with Simultaneous Multi-Slice Acquisition
}

\section{Laurentius Huber ${ }^{a, b^{*}}$, Dimo Ivanov ${ }^{c}$, Maria Guidi ${ }^{a}$, Robert Turner ${ }^{a}$, Kâmil Uludağ ${ }^{c}$, Harald E.} Möller ${ }^{a}$, and Benedikt A. Poser ${ }^{c}$

${ }^{a}$ Max Planck Institute for Human Cognitive and Brain Sciences, Leipzig, Germany

${ }^{b}$ sFIM, NIMH, NIH, Bethesda, MD, USA

${ }^{c}$ Maastricht Brain Imaging Centre, Maastricht University, Maastricht, The Netherlands

*Corresponding author:

Laurentius Huber, PhD

Section on Functional Imaging Methods, NIMH, National Institutes of Health

Building 10, Room 1D80B

10 Center Dr. MSC 1148

Bethesda, MD 20892-1148

Email: Laurentius.Huber@nih.gov

Phone: +1 3014027298

Running Title: CBV mapping with SMS VASO

The body of the text contains 5224 words (with additional 1711 words in references)

Highlights:

- $\quad f M R I$ with CBV-mapping is combined with a simultaneous multi-slice readout.

- FOV is increased up to a factor of 4 to enable near whole-brain coverage.

- High SNR allows high-resolution imaging up to $1 \times 1 \times 1.2 \mathrm{~mm}^{3}$.

- Higher specificity to GM tissue of CBV-fMRI compared to GE-BOLD is confirmed.

\begin{abstract}
The aim of this study is to overcome the current limits of brain coverage available with multi-slice echo planar imaging (EPI) for vascular space occupancy (VASO) mapping. By incorporating simultaneous multi-slice (SMS) EPI image acquisition into slice-saturation slabinversion VASO (SS-SI VASO), many more slices can be acquired for non-invasive functional measurements of blood volume responses.

Blood-volume-weighted VASO and gradient echo blood oxygenation level-dependent (GEBOLD) data were acquired in humans at $7 \mathrm{~T}$ with a 32-channel head coil. SMS-VASO was applied in three scenarios: A) high-resolution acquisition of spatially distant brain areas in the visuo-motor network (V1/V5/M1/S1); B) high-resolution acquisition of an imaging slab covering the entire $\mathrm{M} 1 / \mathrm{S} 1$ hand regions; and $\mathrm{C}$ ) low-resolution acquisition with near wholebrain coverage.

The results show that the SMS-VASO sequence provided images enabling robust detection of blood volume changes in up to 20 slices with signal readout durations shorter than 150 ms. High-resolution application of SMS-VASO revealed improved specificity of VASO to GM
\end{abstract}


tissue without contamination from large draining veins compared to GE-BOLD in the visual cortex and in the sensory-motor cortex.

It is concluded that VASO FMRI with SMS-EPI allows obtaining a reasonable threedimensional coverage not achievable with standard VASO during the short time period when blood magnetization is approximately nulled. Due to the increased brain coverage and better spatial specificity to GM tissue of VASO compared to GE-BOLD signal, the proposed method may play an important role in high-resolution human $\mathrm{FMRI}$ at $7 \mathrm{~T}$.

Abbreviations: $\mathrm{BOLD}=$ blood oxygenation level dependent; $\mathrm{CBV}=$ cerebral blood volume; $\mathrm{CNR}=$ contrast-to-noise ratio; $\mathrm{CSF}=$ cerebrospinal fluid; $\triangle \mathrm{CBV}=$ change in $\mathrm{CBV} ; \mathrm{EPI}=$ echo planar imaging; $\mathrm{fMRI}=$ functional magnetic resonance imaging; $\mathrm{GE}=$ gradient echo; $\mathrm{GM}=$ grey matter; $\mathrm{MT}=$ magnetization transfer; $\mathrm{ROI}=$ region of interest; SNR = signal-to-noise ratio; SS-SI VASO = slice-selective slab-inversion VASO; TE = echo time; $\mathrm{TI}=$ inversion time; $\mathrm{TR}=$ repetition time; VASO = vascular space occupancy.

Keywords: vascular space occupancy, SS-SI VASO, cerebral blood volume, simultaneous multi-slice, multi-band, 7 Tesla MRI

\section{Introduction}

Functional MRI ( $f M R I)$ has revolutionized cognitive neuroscience research. The size of fMRI voxels can now be reduced below one millimeter, approaching the size of individual cortical layers or columns. Since conventional gradient echo (GE) BOLD FMRI measures changes in brain activity only indirectly via blood oxygenation changes (Bandettini, 2012), however, it is limited with respect to its quantifiability and its spatial specificity.

It has been shown (Kim et al., 2013a) that fMRI based on cerebral blood volume (CBV) can map changes of brain activity with better spatial localization than GE-BOLD signal, and without contamination by remote draining veins. The most commonly used method for non-invasive measurements of CBV changes in humans is vascular space occupancy (VASO) (Lu et al., 2003). Similar to CBV mapping in animals based on injection of contrast agents, CBV mapping with VASO in humans provides high functional specificity to activity in neural tissue (Donahue et al., 2006; Huber et al., 2015; Lu et al., 2005). VASO is particularly attractive at high fields $(7 \mathrm{~T})$ due to the increase in image signal-to-noise ratio and the longer blood $T_{1}$ relaxation time, which approaches the vasculature refill time (Huber et al., 2014c). However, VASO requires image acquisition after an inversion pulse at the blood magnetization nulling time of approximately $T_{1} \times \ln (2)$. Thus, if multiple consecutive slices are acquired after each inversion, they end up with different inversion times $(T I)$, limiting the brain coverage of this method. This is especially problematic at high resolutions that require acquisition of relatively long echo trains. 
The acquisition time window and the maximum acquisition duration are usually chosen such that the uncertainty in the blood nulling time $\Delta T_{1} \times \ln (2)$ is smaller than the variation of the imaging time $\Delta T I$ (Lu et al., 2004; Scouten and Constable, 2007). Considering that the variation of blood $T_{1}$ with oxygenation state and haematocrit is in the order of 200 ms (Grgac et al., 2012; Rane and Gore, 2013; Zhang et al., 2012), all VASO data should be acquired within this corresponding time interval. This limits the number of slices that can be acquired with conventional EPI to be not greater than five (Huber et al., 2014a; Huber et al., 2014c; Scouten and Constable, 2007).

Previous studies suggest that VASO coverage can be increased by combining VASO spin preparation with advanced readout techniques such as three-dimensional (3D) GRASE (Donahue et al., 2009; Poser and Norris, 2007, 2009). However, the long acquisition time required for 3D GRASE and the shorter tissue $T_{2}$ at high field strengths can induce through-plane blurring due to $T_{2}$ decay. In high-resolution $f M R I$, the short $T_{2}$ can also introduce limitations with respect to the echo-train length, the number of acquired slices, or spin echo (SE) BOLD signal contamination in VASO. This can be improved by shortening the acquisition window, i.e., by shortening the echo-train length, by means of in-plane parallel imaging. However, the considerable k-space undersampling with high parallel imaging acceleration factors incurs losses in signal-to-noise ratio (SNR). Furthermore, the numerous refocusing radiofrequency (RF) pulses can introduce unacceptable additional energy deposition in sequences limited by specific absorption rate (SAR) restrictions.

Alternatively, VASO coverage can be increased by combining VASO spin preparation with multi-shot 3D readout strategies (Cretti et al., 2013; Hua et al., 2013). However, since in this acquisition scheme different parts of k-space are acquired after separate excitation pulses, only the k-space center is acquired at the blood-nulling time. This implies that the high spatial frequencies (outer $k$-space lines, respectively) will be weighted by blood flow effects in addition to the weighting from blood volume changes, which can introduce inaccuracies in high-resolution fMRI.

Recently, simultaneous multi-slice imaging (SMS) with multi-band (MB) excitation was developed to boost the brain coverage of data acquisition within a given acquisition period (Feinberg et al., 2010; Moeller et al., 2010; Setsompop et al., 2012a). The use of SMS-EPI can multiply the number of acquired slices during the acquisition window in VASO experiments. In addition, it has been shown that the application of SMS-EPI can be advantageous to overcome brain coverage limitations in ASL (Feinberg et al., 2013; Ivanov et al., 2014; Kim et al., 2013b; Wang et al., 2015).

The purpose of this study is to implement and evaluate high-resolution functional imaging with VASO and BOLD contrast using SMS-EPI for concurrent imaging of CBV and oxygenation level. 
This study focuses on the most important limitations of VASO-fMRI: the challenges to simultaneously acquire enough imaging slices to cover multiple brain areas, and to increase the slice resolution within an extended brain region. The novel VASO sequence is applied in three experimental setups: A) high-resolution acquisition of distant brain areas in the visuo-motor network (V1/V5/M1/S1); B) high-resolution acquisition of an imaging slab covering the entire $\mathrm{M} 1 / \mathrm{S} 1$ hand region; C) low-resolution acquisition with near to whole-brain coverage.

\section{Materials and Methods}

\subsection{MR sequence}

MRI data were acquired with ten healthy right-handed participants (age 22-29 years). All procedures were approved by the Ethics Committee of the University of Leipzig. Informed written consent was given by all volunteers. SMS-SS-SI VASO was implemented on a MAGNETOM 7T scanner (Siemens Healthcare, Erlangen, Germany) in IDEA. For RF transmission and reception, a single-channel-transmit/32-channelreceive head coil (Nova Medical, Wilmington, MA, USA) was used. Functional data were acquired using an SMS two-dimensional (2D) single-shot EPI readout. The timing of magnetization preparation and interleaved acquisition of VASO and BOLD data is schematically depicted in Fig. 1 . Sequence parameters were: $T I 1 / T 12 / T R=$ $1100 / 2600 / 3000 \mathrm{~ms}$, nominal excitation flip angle was $\alpha=70^{\circ}-90^{\circ}$ dependent on the head size and the corresponding variation of the transmit voltage within SAR limits, across all functional experiments. The summation of multi-band sinc-pulses was conducted with optimized phase schedules for minimizing peak RF power (Wong, 2012). VASO can be contaminated by inflow of non-inverted, especially, when blood $T_{1}$ is not much shorter than TR (Donahue et al., 2006). Such inflow effects can be avoided, when the blood-nulling time is shorter than the time that blood needs to arrive from the arteries in the neck to the micro vessels of the imaging slice (Huber et al., 2014). Here, a $T / 1=1100 \mathrm{~ms}$ is chosen, which includes an additional leeway of 200 ms compared to the estimated arterial arrival time in the sensorimotor cortex (Mildner et al., 2014). The blood nulling time was manipulated by means of an adjusted inversion efficiency of $87 \%$ in a $\mathrm{B}_{1}$-independent manner by using a phase skip of the RF field during inversion as described in (Huber et al., 2014c). The inversion pulse shapes are based on the TR-FOCI pulse (Hurley et al., 2010). The efficiency of the pulse was $94 \%$ (measured in pilot experiments). This means that $6 \%$ of the magnetization is lost during the application of the pulse. The inversion pulse amplitude was adjusted to have a minimum of $10 \mu \mathrm{T}$ down to the Circle of Willis across all participants by used a transmitter voltage of $340 \mathrm{~V}$. The overall energy deposition of the sequence never exceeded $2.1 \mathrm{~W} / \mathrm{kg}$, according to the SAR estimation of the vendor. The blood nulling time is calculated based on the assumed value of 
blood $T_{1}=2100 \mathrm{~ms}$, following earlier VASO studies at $7 \mathrm{~T}$ (Huber et al., 2014a; Huber et al., 2015; Huber et al., 2014c).

With increasing field strength, the positive BOLD signal change during neural activation increasingly counteracts the negative VASO signal change. The GE-BOLD effect typically has two components: intra-vascular and extravascular. At $7 \mathrm{~T}$, the extravascular BOLD dominates the intravascular BOLD by more than $90 \%$ (Donahue et al., 2010; Uludağ et al., 2009). This extravascular BOLD contamination is considerably larger than the desired VASO signal change and needs to be corrected for. In SS-SI VASO, an interleaved, pair-wise acquisition of VASO and BOLD images is used to distinguish between BOLD and VASO signal components of the resulting signal. When the pure BOLD contrast contribution is known, the BOLD-contamination in the VASO image can be factored out, as described earlier (Huber et al., 2014a). In short, both BOLD and VASO time series are expected to have the same BOLD $T_{2}{ }^{*}$-weighting but different VASO $T_{1}$ weighting. Hence, when the voxel-wise ratio image between the BOLD and the VASO images is formed, the $T_{2}{ }^{*}$-weighting is canceled out. This provides a BOLD-corrected VASO contrast. As long as both images are acquired with the identical EPI module, the $T_{2}{ }^{*}$-weighting cancels out, independent of TE and readout duration. This BOLD correction mechanism relies on the assumption that changes in BOLD weighting are slower than the time between consecutive image acquisitions $(3 \mathrm{~s}$ for a pair of VASO and BOLD). This means that any temporal dynamics of the BOLD signal change faster than $3 \mathrm{~s}$ will not be corrected for. Furthermore, it assumes that extravascular effects contribute much more to the BOLD response than intravascular effects. In this correction scheme, BOLD contaminations are considered to be solely based on changes in $T_{2}{ }^{*}$. Hence, the applied correction scheme is assumed to account for BOLD contaminations in all compartments of the vascular tree including arteries, capillaries and veins inside and outside the GM tissue. This means that the BOLDcorrection is not expected to have any limitations with respect to spatial resolution.

All functional experiments consisted of one-minute blocks repeated 12 times. The VASO sequence parameters concerning resolution, acceleration and position were optimized for three specific cases described as follows:

\subsubsection{Experiment A: Two high-resolution slice groups}

Two slice groups were positioned to cover V1/V5 and M1/S1 in 6 out of the 10 participants, using the following sequence parameters: nominal resolution = $0.97 \times 0.97 \times 1.1 \mathrm{~mm}^{3}-1 \times 1 \times 1.5 \mathrm{~mm}^{3}$, depending on the participant's brain anatomy; no slice gaps; $2 \times 5=10$ slices; SMS factor $=2$; field-of-view (FoV) shift (CAIPI factor) $=1$; GRAPPA factor $=3$, segmented reference line acquisition; $T E$ = $34 \mathrm{~ms}$. The maximal time difference of image acquisition to the blood nulling time $\Delta T /$ was $162 \mathrm{~ms}$. This means that the first excitation pulse was applied 162 
ms before the blood nulling time point and the last excitation pulse was applied $162 \mathrm{~ms}$ after the blood nulling time point as indicated in Fig. 1. The distance between the centers of the two slice groups was $14-17 \mathrm{~mm}$, depending on tilting angle and the participants' anatomy. This distance refers to the gap between the two yellow imaging slabs depicted in Fig. 2A. Partial Fourier imaging was kept minimal with a factor of $=7 / 8$. The fat saturation pulse flip angle was set to $30^{\circ}$, assuming that most of the fat signal due to its short $T_{2}$ would be decayed during the EPI readout anyway, not leading to serious fat artifacts. The functional paradigm consisted of three conditions: 20s rest, 20s visual task with a high-contrast static star field, and $20 \mathrm{~s}$ visual task with a highcontrast moving star field as in (Huk et al., 2002) with concurrent finger motion during the star motion. The finger motion consisted of pinch-like movement and touch of index finger, middle finger, ring finger, and little finger (consecutively) towards and away from the thumb with a self-paced frequency of approximately $0.25-0.75 \mathrm{~Hz}$.

\subsubsection{Experiment $B$ : Whole $M 1$ coverage with high spatial resolution}

One slice group was positioned to cover the entire areas of M1/S1 in 6 out of the 10 participants, with the following sequence parameters: nominal resolution = $1 \times 1 \times 1.2 \mathrm{~mm}^{3}-1 \times 1 \times 1.5 \mathrm{~mm}^{3}$, depending on the participant's brain anatomy; 50\% - 70\% slice gap; 15 slices; maximal $\Delta T / 1=175 \mathrm{~ms}$; SMS factor $=3$; FoV shift $=1 / 3$; GRAPPA factor $=2$ with FLEET (Chapman et al., 1987) reference lines (Polimeni et al., 2015); $T E=33 \mathrm{~ms}$. To minimize $T_{2}{ }^{*}$-blurring, partial Fourier imaging was not used (Huber et al., 2014b). The functional paradigm consisted of unilateral finger-tapping (alternating 30-s rest vs. 30-s tapping). The tapping task was identical to experiment $A$.

\subsubsection{Experiment C: Near whole-brain coverage with low spatial resolution}

20 slices were positioned to cover the brain in 6 out of the 10 participants with the sequence parameters: nominal resolution $=3 \times 3 \times 3 \mathrm{~mm}^{3} ; 50 \%$ slice gaps; maximal, $\Delta T I 1=75 \mathrm{~ms}$; SMS factor $=4 ;$ FoV shift factor $=1 / 3 ;$ GRAPPA factor $=2$ with FLEET reference lines; $T E=14 \mathrm{~ms}$, partial Fourier factor $=7 / 8$. The functional paradigm was identical to that of experiment $A$.

\subsubsection{Direct comparison of SMS-VASO with non-SMS-VASO}

In order to evaluate, whether the application of SMS imaging in VASO may degrade the image quality, direct comparisons of SMS-VASO and non-SMSVASO were conducted. In three additional participants, the imaging protocols described above (experiments $\mathrm{A}-\mathrm{C}$ ) were repeated without functional stimulation along with a variant with SMS factor 1 (non-SMS) leaving all other parameters the same. With the reduced acceleration in the non-SMS-VASO 
case, only $20 \%$ to $50 \%$ of the imaging region could be covered compared to SMS-VASO. Hence, not all ROIs could be compared (V1, V5, M1/S1). Here, the sensory motor area was chosen as a region for comparison. l.e. the imaging slices were positioned to cover the M1/S1 area with both protocols, with the SMS-VASO and the non-SMS-VASO.

Note that experiment $A$ uses the segmented GRAPPA reference line acquisition, while experiment $B$ and $C$ use FLEET GRAPPA reference line acquisition. The GRAPPA reference line acquisition scheme of choise was decided based on respective susceptibility to $B_{0}$-inhomogenieties, appearance of GRAPPA-ghosts and tSNR obtained in respective pilot experiments.

\subsection{Image reconstruction}

Image reconstruction was performed online on the scanner. The data with $7 / 8$ partial Fourier were zero-filled. Signals from the simultaneously acquired slices were first unaliased with an implementation of SplitSlice-GRAPPA with LeakBlock (Cauley et al., 2014) and a $3 \times 3$ kernel, as distributed with the MGH blipCAIPI C2P (Setsompop et al., 2012b)(http://www.nmr.mgh.harvard.edu/software/c2p/sms); this was followed by the vendor's in-plane GRAPPA reconstruction (Griswold et al., 2002), using a $2 \times 3$ kernel. Finally, the complex coil images were combined using the vendor's implementation of adaptive combine.

\subsection{Data analysis}

All MR images were motion corrected using SPM8 (Wellcome Department, University College London, UK). Statistical analysis was done using FSL FEAT (Version 5.98) (Worsley, 2001). One of the major goals of this study is to investigate the spatial features of CBV and BOLD signal changes in brain areas that are already known to be involved in the functional task. Therefore, the purpose of the statistical analysis is not to isolate significantly activated areas from other brain areas, but to investigate the range of $Z$-values within the ROIs. Hence, $Z$-value thresholds in statistical activation maps are kept relatively low, minimizing false negative voxels despite increasing the risk of false positive voxels. Statistical data were not thresholded by cluster size. In order to estimate and eliminate BOLD contamination in the CBV-weighted data, the VASO signal was corrected using the BOLD signal acquired interleaved, as in previous studies (Huber et al., 2014c). In the evaluation of data from experiment B (thick highresolution imaging slab covering $\mathrm{M} 1$ ), missing signal from inter-slice gaps was linearly extrapolated from adjacent slices to provide undistorted signal maps in the coronal and sagittal orientations. No spatial smoothing was applied during any part of the offline data analysis.

\section{Results}




\subsection{Experiment A: Two slice groups covering V1, V5, M1, and S1}

The results of SMS-VASO imaging of the visuo-motor network are depicted in Fig. 2. Maps of temporal SNR (tSNR) are depicted in Fig. 2A for one representative subject. It can be seen that the proposed method has enough tSNR to significantly detect strong activity changes in V1 and in the contralateral sensory motor cortex. The inter-subject average tSNR of the VASO and BOLD signal in grey matter (GM) ROIs is $16 \pm 3$ and $22 \pm$ 4 , respectively. Indications of positive and negative activity changes in the ipsilateral sensory-motor region and in V5 are clearly visible, but not as robustly as in the contralateral sensory-motor region.

The contralateral M1 and S1 yielded significant signal increases during the unilateral finger motion task. The ipsilateral M1 shows a positive BOLD signal change and CBV increase, while ipsilateral S1 shows a negative BOLD and CBV decrease in all participants.

Black arrows in the lower slice of the results in Fig. 2A refer to fat signal artifacts, which most probably result from insufficient fat suppression. Since they are expected to be the same at every time point during the experiment, they can be considered negligible with respect to the functional results presented.

Fig. 2B depicts the reproducibility and stability of the results across participants. The average signal changes of the contralateral BOLD and VASO responses were $(2.3 \pm$ $0.5) \%$ and $(-1.7 \pm 0.5) \mathrm{ml} / 100 \mathrm{ml}$ respectively in $\mathrm{M} 1$ and $(2.9 \pm 0.4) \%$ and $(2.0 \pm 0.5)$ $\mathrm{ml} / 100 \mathrm{ml}$ in S1, respectively. The average signal changes of the ipsilateral BOLD and VASO responses were $(1.8 \pm 0.5) \%$ and $(-1.1 \pm 0.4) \mathrm{ml} / 100 \mathrm{ml}$, respectively in $\mathrm{M} 1$ and $(-1.6 \pm 0.4) \%$ and $(1.2 \pm 0.4) \mathrm{ml} / 100 \mathrm{ml}$ in $\mathrm{S} 1$, respectively.

It is worth pointing out that BOLD is inherently sensitive to task-correlated motion (Fig. 2; green arrows in participants 1 and 2) (Schulz et al., 2014), while any variations in the signal beyond functional $T_{1}$ changes are inherently suppressed in the BOLDcorrection procedure (which involves taking the ratio of sequential images) in SS-SI VASO.

The average time courses of GE-BOLD and VASO response signals are depicted in Fig. 2C. V1 is sensitive to contrast changes, and is largely independent of the amount of motion in the visual presentation. V5, contralateral $M 1$, contralateral S1, and ipsilateral M1 have an overall activation response during movements, while ipsilateral S1 has an overall deactivation response during unilateral movements. This is consistent with CBV and BOLD signal responses in previous studies (Huber et al., 2015). Following the BOLD and VASO time courses, it can be seen that there is no significant post-stimulus undershoot, even in V1. This is consistent with previous BOLD and VASO results using the same visual moving star field paradigm (Huber et al., 2014c), which shows a slightly different response shape compared to a more common 
flickering checkerboard paradigm. It must be also noted that the occurrence of a significant post-stimulus undershoot is highly dependent on the extent of the area of interest (van Zijl, et al., 2012), and the inter-stimulus resting period used (Huber et al., 2014d).

\subsection{Experiment B: High-resolution M1 coverage}

The results of SMS-VASO for high-slice-resolution imaging of an imaging slab spanning across M1 are depicted in Fig. 3. The average tSNR (Fig. 3A) of VASO and BOLD signal in GM ROls of M1/S1 is $14 \pm 2$ and $24 \pm 4$, respectively. The tSNR is sufficient to obtain highly consistent and reproducible results across participants (Fig. 3B). The activity pattern in contralateral and ipsilateral sensory motor cortex is very similar to the results from experiment $A$ (Fig. 2). 


\section{Experiment C: Near whole-brain coverage acquisition}

The results of SMS-VASO for low-resolution aqusisitions to cover nearly the entire brain are depicted in Fig. 4. Average tSNR (Fig. 4A) of VASO and BOLD signal in GM ROIs is $33 \pm 6$ and $57 \pm 12$, respectively. The activity patterns are very similar across participants (Fig. 4B), and they are consistent with the results of experiment A (Fig. 2). The limited robustness in the detection of small negative response in ipsilateral S1 and the small positive response in V5 might be a result of the partial voluming of GM with white matter and cerebro-spinal fluid (CSF), or partial voluming of opposite responses in $\mathrm{M} 1 / \mathrm{S} 1$ at low resolution.

\subsection{Direct comparison of SMS-VASO with non-SMS-VASO}

The results of the direct comparison of SMS-VASO and non-SMS-VASO are depicted in Fig. 5. The depicted tSNR maps show that there is no significant image quality reduction, when applying SMS-VASO compared to non-SMS-VASO. The tSNR in the M1/S1 region for SMS-VASO and non-SMS VASO was $18 \pm 4$ and $19 \pm 4$ for experiment A, $15 \pm 3$ and $14 \pm 4$ for experiment $B$, and $33 \pm 5$ and $29 \pm 4$ for experiment $C$, respectively. The negligible tSNR difference with and without SMS imaging is consistent with previous studies. Setsompop et al. showed that the g-factor remains around 1.0 for SMS imaging with CAIPI and SMS factors up to 3 (Setsompop, et al., 2012).

\subsection{Summary of statistical numerical results of functional results}

The statistical Z-values within activated regions depicted in Figs 2-4 are summarized in Tab. 1 . The manually selected ROIs are V1, V5, and the contralateral side of M1. It can be seen that the statistical Z-values are larger for BOLD compared to VASO. This is consistent with the higher TSNR in BOLD compared to VASO.

\section{Discussion}

The results shown in Figs. 2 and 3 clearly demonstrate a major advantage of high-resolution VASO. This methodology can distinguish different individual responses in neighboring but distinct brain areas (e.g. ipsilateral S1 and M1), that cannot be separated with GE-BOLD, with low-resolution fMRI, or when applying spatial smoothing (Stelzer et al., 2014).

\subsection{Other 3D imaging approaches}

Besides the combination of VASO with SMS acquisition, several advanced imaging strategies have been proposed to increase the coverage of VASO (Lu et al., 2013). These include MAGIC VASO (Lu et al., 2004), 3D GRASE VASO (Donahue et al., 2009; Poser and Norris, 2009), HASTE VASO (Poser and Norris, 2007), multi-shot 3D turbo field echo VASO (Hua et al., 2013), and multi-shot 3D TSE VASO (Cretti et al., 2013). Compared with these previously suggested approaches for increasing the VASO 
coverage, the proposed SMS acquisition is particularly beneficial for obtaining high spatial resolution, because it can increase the coverage without increasing the acquisition duration. For a further increase in SMS-VASO coverage, the technique could be combined with the MAGIC VASO (Lu et al., 2004) approach, in which the blood signal is forced to pass through zero multiple times by means of additional inversion pulses during the acquisition. However, the SAR constraints of the corresponding additional inversion pulses at high field strengths might limits its application dependent on the efficiency of the hardware available. More research is needed to determine the applicability of MAGIC VASO at high field strengths.

\subsection{Limitation by $T R$ for vasculature refilling}

The proposed high tSNR of SS-SI VASO compared to the traditional VASO approach is based on additional assumptions regarding the blood flow dynamics. For complete nulling of once-inverted blood magnetization in SS-SI VASO, it is required that all the blood within the imaging slice is refilled within one TR. It is estimated in the original SS-SI VASO paper (Huber et al., 2014c) that it takes 1-1.5 s until the microvasculature of a single slice is refilled with fresh blood. For a thicker imaging slab, the refilling time is expected to be correspondingly longer. In order to avoid incomplete blood nulling in the proposed SMS-SS-SI VASO method, we chose the TR to be minimally $3 \mathrm{~s}$, giving the blood enough time to refill the entire brain. With this sequence timing, the measured changes in V1 are not different from the estimated changes in V1 in previous studies, acquired with the same activation task but a single-slice implementation (Huber et al., 2014c). This suggests that with the sequence timing used in this study, the acquisition of more slices does not lead to additional violation of the refill condition.

\subsection{Effect of incomplete blood nulling}

There are two major sources of incomplete blood nulling in VASO. (i) Uncertainties in blood $T_{1}$, e.g. due to physiologic reasons, such as oxygenation level and inter-subject variations in hematocrit. (ii) Variations in T/1, due to technical reasons, e.g. the consecutive acquisition of multiple $2 \mathrm{D}$ slices. It has been recently shown that the difference between arterial blood $T_{1}$ and venous blood $T_{1}$, and the influence of moderate variations in hematocrit lies in the range of $100-200 \mathrm{~ms}$ (Grgac et al., 2012; Rane and Gore, 2013). A sequence of up to five consecutive excitation pulses causes a variation in blood-nulling time of the same order (75- $175 \mathrm{~ms})$. The corresponding incomplete blood nulling can result in an error in the VASO signal change of up to $14 \%$ relative to the total VASO signal change. This means that in the worst-case scenario, the measured CBV change of $2.0 \pm 0.5 \mathrm{ml} / 100 \mathrm{ml}$ in contralateral $\mathrm{M} 1$ might have an additional source of uncertainty, to become $2.0 \pm 0.5$ (inter-subject standard deviation) \pm 0.28 (uncertainty in blood-nulling time) $\mathrm{ml} / 100 \mathrm{ml}$. Since the corresponding bias of VASO quantification from slices acquired before blood nulling and from slices acquired after blood nulling is opposite in sign, these biases are 
believed to largely cancel each other out after averaging across ROls and, thus, to have no significant effect on the averaged results of this study.

\subsection{Signal change at cortical surface}

The VASO contrast at the cortical surface can suffer from artifacts arising from (i) BOLD contaminations and (ii) dynamic changes in CSF volume (Lu et al., 2013) that could complicate the interpretation of CBV changes at the cortical surface. While conventional VASO contrast generation can suffer from these contaminations, they have only a limited effect in the application of SS-SI VASO, as discussed in (Huber et al., 2015). (i) Any extravascular BOLD contamination is corrected for in SS-SI VASO by means of dynamic division by the interleaved-acquired BOLD signal. (ii) Contamination of dynamic changes in partial volume from CSF can be minimized in SS-SI VASO by manipulation of the steady-state CSF magnetization such that it has a positive phase and a similar signal compared to GM. Hence, it is expected that the high GM tissue specificity of SS-SI VASO is dominated by the sensitivity to microvascular vessels.

In two out of six participants, there are a few voxels clearly between the two sides of the contralateral sulcus that show a positive VASO response, suggesting vasoconstriction (participant 2 and 4 in Fig. 3). Such features have also been reported by others, and have been interpreted as volume constriction of large draining veins (Blockley et al., 2012) or neural inhibition (Trampel et al., 2013).

\subsection{Functional specificity}

Data given in Figs. 2 and 3 show that VASO fMRI can better delineate individual GM territories, as compared with GE-BOLD which invariably shows largest activity between the opposing GM banks of a sulcus. The higher spatial specificity of VASO to GM tissue without contamination from independent of large draining veins can be particularly rewarding when opposing GM banks of a sulcus comprise different nodes of a brain network (e.g. M1 and S1). Where there is positive response in opposite GM areas (e.g. contralateral M1/S1), the GE-BOLD signal of both areas is amplified by the draining vein effect (Turner, 2002) to be maximal in larger veins above the cortical surface (see purple inserts in Figs. 2 and 3 ).

In the case of positive and negative responses in opposing GM banks (e.g. ipsilateral $\mathrm{M} 1 / \mathrm{S} 1$ ), the mixing of deoxyhemoglobin changes arising from opposite responses on opposite sides of the sulcus can result in an attenuated GE-BOLD signal (see blue inserts in Figs. 2 and 3). For example, pial veins within the sulcus can drain both M1 and $\mathrm{S} 1$, and hence their BOLD signal might reflect a mixture of activity in both areas. Such features, which have not previously been discussed in the literature, can make it difficult to interpret the corresponding BOLD signal from opposite sides of the sulcus. The higher specificity to brain tissue in VASO, avoids such limitations, and depicts responses in opposing GM banks of the central sulcus independently. 


\subsection{Response in the ipsilateral hemisphere}

Figs. 2 and 3 show that unilateral finger movement evokes a positive response in ipsilateral M1, but a negative response in ipsilateral S1. This particular result has been found to be highly dependent on stimulus paradigm and strength. While low force (usually $5 \%$ of individual maximal voluntary contraction) has been shown to evoke a negative BOLD response, and reductions in blood flow and metabolism in ipsilateral sensori-motor ROls, positive responses in ipsilateral M1 have been observed when stronger forces and more demanding tasks are used (Dettmers et al., 1995).

\subsection{Magnetization transfer effects in SMS-VASO}

The application of off-resonant RF pulses in inversion recovery sequences such as VASO, results in magnetization transfer (MT) effects. These MT effects can result in an accelerated longitudinal relaxation mimicking a different $T_{1}$. In the application of SMSVASO the (off-) resonant excitation pulses have increased RF amplitude and thus also higher potential to induce MT effects. While there are significant MT effects in most brain tissues, it has been shown that blood exhibits a very small MT effect due to its low concentration of macromolecules (Wolff and Balaban, 1989; Balaban et al., 1991). This means that while off-resonant RF pulses affect the tissue relaxation, blood relaxation is not altered, leaving the blood nulling time unaffected. In fact, this unique difference between blood and brain tissue regarding MT effects has been used to actively enhance SNR in high field VASO experiments by means of additional highpower MT pulses (Hua et al., 2009 and 2013). In conclusion, the additional MT effects due to the multiband excitation pulses do not alter the blood nulling time and CBV quantification, but are believed to result in a slightly larger GM signal and corresponding SNR increase.

\subsection{Extensibility to lower field strengths}

While all experiments in this study were conducted at $7 \mathrm{~T}$, its application might be advantageous at $3 \mathrm{~T}$ as well. The shorter blood $T_{1}$ at lower field strengths (Zhang et al., 2013), however, results in reduced signal gain applying SS-SI VASO as opposed to the original VASO (Huber et al., 2014c). Additionally, the larger relative thermal noise contribution at $3 \mathrm{~T}$ compared to $7 \mathrm{~T}$ can also be an additional constraint in going to such high spatial resolutions at lower field strengths. The low-resolution approach of experiment $C$, however, might be a useful tool for quantitative $\mathrm{fMRI}$ techniques, such as calibrated BOLD, both at $3 \mathrm{~T}$ and $7 \mathrm{~T}$. More research is necessary to evaluate the combination of VASO and SMS imaging at $3 \mathrm{~T}$.

\subsection{Other imaging modalities}

Spin echo (SE) BOLD fMRI has been suggested to have higher specificity to the microvasculature (Uludağ et al., 2009) and its utility for laminar and columnar fMRI 
has been demonstrated in animals (Goense et al., 2012; Harel et al., 2006; Zhao et al., 2006) and in humans (Yacoub et al., 2005; Yacoub et al., 2008). However, it suffers from much lower sensitivity, especially at high resolution (Yacoub et al., 2005), which may limit the widespread application of the technique (Boyacioglu et al., 2014; Budde et al., 2014; Harmer et al., 2012).

\section{Conclusion}

SMS-EPI has a major advantage in VASO FMRI by acquisition of more slices during the short time period $\Delta T I$ when blood magnetization is sufficiently nulled. Due to the increased brain coverage and better localization specificity of VASO to GM tissue compared to GE-BOLD signal, the proposed method can play an important role in highresolution $\mathrm{fMRI}$ at $7 \mathrm{~T}$.

\section{Acknowledgements}

We thank Domenica Wilfling and Elisabeth Wladimirow for radiographic assistance. We thank Steve Cauley at MGH for sharing the interface of their image reconstruction for use with our SMS-VASO sequence. The research was supported by the Max Planck Society and Netherlands Organization for Scientific Research NWO: VIDI 452-11-002 to Kamil Uludağ. Maria Guidi was supported by the Initial Training Network, HiMR, funded by the FP7 Marie Curie Actions of the European Commission (FP7-PEOPLE2012-ITN-316716). Preliminary accounts of this work have been presented in the Proceedings of the 23th Annual Meeting of ISMRM, Toronto, Canada, 2015 (abstract $600)$.

\section{References}

Balaban R.S., Chesnick S., Hedges K., Samaha F., Heineman F.W. Magnetization transfer contrast in MR imaging of the heart. Radiology 1991; 180:671-675.

Bandettini, P.A., 2012. Twenty years of functional MRI: the science and the stories. Neuroimage 62, 575-588.

Blockley, N.P., Driver, I.D., Fisher, J.A., Francis, S.T., Gowland, P.A., 2012. Measuring venous blood volume changes during activation using hyperoxia. Neuroimage 59, 3266-3274.

Boyacioglu, R., Schulz, J., Muller, N.C., Koopmans, P.J., Barth, M., Norris, D.G., 2014. Whole brain, high resolution multiband spin-echo EPI fMRI at $7 \mathrm{~T}$ : A comparison with gradient-echo EPI using a color-word Stroop task. Neuroimage 97, 142-150.

Budde, J., Shajan, G., Zaitsev,M., Scheffler, K., Pohmann, R., 2014. FunctionalMRI in human subjects with gradient-echoandspin-echo EPI at 9.4T.Magn. Reson.Med. 71, 209-218.

Cauley, S.F., Polimeni, J.R., Bhat, H., Wald, L.L., Setsompop, K., 2014. Interslice leakage artifact reduction technique for simultaneous multislice acquisitions. Magn Reson Med 72, 93-102.

Chapman, B., Turner, R., Ordidge, R.J., Doyle, M., Cawley, M., Coxon, R., Glover, P., Mansfield, P., 1987. Real-time movie imaging from a single cardiac cycle by NMR. Magn Reson Med 5, 246254. 
Cretti, F.R., Summers, P.E., Porro, C.A., 2013. Multi-shot turbo spin-echo for 3D vascular space occupancy imaging. Magn Reson Imaging 31, 875-881.

Dettmers, C., Fink, G.R., Lemon, R.N., Stephan, K.M., Passingham, R.E., Silbersweig, D., Holmes, A., Ridding, M.C., Brooks, D.J., Frackowiak, R.S., 1995. Relation between cerebral activity and force in the motor areas of the human brain. J Neurophysiol 74, 802-815.

Donahue, M.J., Hoogduin, H., van Zijl, P.C.M., Jezzard, P., Luijten, P.R., Hendrikse, J., 2010 Blood oxygenation level-dependent (BOLD) total and extravascular signal changes and $\triangle R 2 *$ in human visual cortex at 1.5, 3.0 and 7.0 T. NMR Biomed 24, 25-34.

Donahue, M.J., Blicher, J.U., Ostergaard, L., Feinberg, D.A., MacIntosh, B.J., Miller, K.L., Gunther, M., Jezzard, P., 2009. Cerebral blood flow, blood volume, and oxygen metabolism dynamics in human visual and motor cortex as measured by whole-brain multi-modal magnetic resonance imaging. J Cereb Blood Flow Metab 29, 1856-1866.

Donahue, M.J., Lu, H., Jones, C.K., Edden, R.A., Pekar, J.J., van Zijl, P.C., 2006. Theoretical and experimental investigation of the VASO contrast mechanism. Magn Reson Med 56, 12611273.

Feinberg, D.A., Beckett, A., Chen, L., 2013. Arterial spin labeling with simultaneous multi-slice echo planar imaging. Magn Reson Med 70, 1500-1506.

Feinberg, D.A., Moeller, S., Smith, S.M., Auerbach, E., Ramanna, S., Gunther, M., Glasser, M.F., Miller, K.L., Ugurbil, K., Yacoub, E., 2010. Multiplexed echo planar imaging for sub-second whole brain FMRI and fast diffusion imaging. PLoS One 5, e15710.

Goense, J., Merkle, H., Logothetis, N.K., 2012. High-resolution fMRI reveals laminar differ- ences in neurovascular coupling between positive and negative BOLD responses. Neuron 76, 629-639.

Grgac, K., van Zijl, P.C., Qin, Q., 2012. Hematocrit and oxygenation dependence of blood (1) $H(2) O$ $\mathrm{T}(1)$ at 7 tesla. Magn Reson Med 70, 1153-1159.

Griswold, M.A., Jakob, P.M., Heidemann, R.M., Nittka, M., Jellus, V., Wang, J., Kiefer, B., Haase, A., 2002. Generalized autocalibrating partially parallel acquisitions (GRAPPA). Magn Reson Med 47, 1202-1210.

Harel, N., Lin, J., Moeller, S., Ugurbil, K., Yacoub, E., 2006. Combined imaging-histological study of cortical laminar specificity of fMRI signals. Neuroimage $29,879-887$.

Harmer, J., Sanchez-Panchuelo, R.M., Bowtell, R., Francis, S.T., 2012. Spatial location and strength of BOLD activation in high-spatial-resolution $\mathrm{FMRI}$ of the motor cortex: a comparison of spin echo and gradient echo fMRI at 7 T. NMR Biomed. 25, 717-725.

Hua J., Donahue M.J., Zhao J.M., Grgac K., Huang A.J., Zhou J., van Zijl P.C. Magnetization transfer enhanced vascular-space-occupancy (MT-VASO) functional MRI. Magn Reson Med 2009;61:944.

Hua, J., Jones, C.K., Qin, Q., van Zijl, P.C., 2013. Implementation of vascular-space-occupancy MRI at 7T. Magn Reson Med 69, 1003-1013.

Huber, L., Goense, J., Kennerley, A.J., Ivanov, D., Krieger, S.N., Lepsien, J., Trampel, R., Turner, R., Moller, H.E., 2014a. Investigation of the neurovascular coupling in positive and negative BOLD responses in human brain at 7 T. Neuroimage 97, 349-362.

Huber, L., Goense, J., Kennerley, A.J., Trampel, R., Guidi, M., Reimer, E., Ivanov, D., Neef, N., Gauthier, C.J., Turner, R., Moller, H.E., 2015. Cortical lamina-dependent blood volume changes in human brain at $7 \mathrm{~T}$. Neuroimage 107, 23-33.

Huber, L., Guidi, M., Goense, J., Mildner, T., Trampel, R., Schulz, J., Eichner, C., Turner, R., Möller, H.E., 2014b. The magnitude point spread function is an inadequate measure of T2*-blurring in EPI. Proceedings of the International Society of Magnetic Resonance in Medicine 23, 2056.

Huber, L., Ivanov, D., Krieger, S.N., Streicher, M.N., Mildner, T., Poser, B.A., Moller, H.E., Turner, R., 2014c. Slab-selective, BOLD-corrected VASO at 7 Tesla provides measures of cerebral blood volume reactivity with high signal-to-noise ratio. Magn Reson Med 72, 137-148. 
Huber, L., Kennerley, A.J., Ivanov, D., Gauthier, GJ., Moeller, H.E., Turner, R., 2014c, Trial-wise investgation of cerebral blood volume change in human brain at 7T, Proceedings of the International Society of Magnetic Resonance in Medicine 22, 3095.

Hurley, A.C., Al-Radaideh, A., Bai, L., Aickelin, U., Coxon, R., Glover, P., Gowland, P.A. Tailored RF pulse for magnetization inversion at ultrahigh field. Magn Reson Med. 63, 51-58

Huk, A.C., Dougherty, R.F., Heeger, D.J., 2002. Retinotopy and functional subdivision of human areas MT and MST. J. Neurosci. 22, 7195-7205.

Ivanov, D., Poser, B.A., Huber, L., Pfeuffer, J., Uludag, K., 2014. Whole-brain perfusion measurements at 7T using pulsed arterial spin labelling and simultaneous multi-slice multi-echo echo planar imaging. Proceedings of the International Society of Magnetic Resonance in Medicine 22, 2698.

Kim, S.G., Harel, N., Jin, T., Kim, T., Lee, P., Zhao, F., 2013a. Cerebral blood volume MRI with intravascular superparamagnetic iron oxide nanoparticles. NMR Biomed 26, 949-962.

Kim, T., Shin, W., Zhao, T., Beall, E.B., Lowe, M.J., Bae, K.T., 2013b. Whole brain perfusion measurements using arterial spin labeling with multiband acquisition. Magn Reson Med 70, 1653-1661.

Lu, H., Donahue, M.J., Jones, C.K., van Zijl, P.C., 2005. Spatial characteristics of VASO fMRI at ultra-high resolution. Proceedings of the International Society of Magnetic Resonance in Medicine 22, 0027.

Lu, H., Golay, X., Pekar, J.J., van Zijl, P.C.M., 2003. Functional magnetic resonance imaging based on changes in vascular space occupancy. Magn Reson Med 50, 263-274.

Lu, H., Hua, J., van Zijl, P.C., 2013. Noninvasive functional imaging of cerebral blood volume with vascular-space-occupancy (VASO) MRI. NMR Biomed 26, 932-948.

Lu, H., van Zijl, P.C., Hendrikse, J., Golay, X., 2004. Multiple acquisitions with global inversion cycling (MAGIC): A multislice technique for vascular-space-occupancy dependent fMRI. Magn Reson Med 51, 9-15.

Mildner, T., Muller, K., Hetzer, S., Trampel, R., Driesel, W., Moller, H.E., 2014. Mapping of arterial transit time by intravascular signal selection. NMR Biomed 27, 594-609.

Moeller, S., Yacoub, E., Olman, C.A., Auerbach, E., Strupp, J., Harel, N., Ugurbil, K., 2010. Multiband multislice GE-EPI at 7 tesla, with 16-fold acceleration using partial parallel imaging with application to high spatial and temporal whole-brain fMRI. Magn Reson Med 63, 1144-1153.

Polimeni, J.R., Bhat, H., Witzel, T., Benner, T., Feiweier, T., Inati, S.J., Renvall, V., Heberlein, K., Wald, L.L., 2015. Reducing sensitivity losses due to respiration and motion in accelerated echo planar imaging by reordering the autocalibration data acquisition. Magn Reson Med.

Poser, B.A., Norris, D.G., 2007. Measurement of activation-related changes in cerebral blood volume: VASO with single-shot HASTE acquisition. Magn Reson Mater Phy 20, 63-67.

Poser, B.A., Norris, D.G., 2009. 3D single-shot VASO using a Maxwell gradient compensated GRASE sequence. Magn Reson Med 62, 255-262.

Rane, S.D., Gore, J.C., 2013. Measurement of T1 of human arterial and venous blood at 7T. Magn Reson Imaging 31, 477-479.

Schulz, J., Siegert, T., Bazin, P.L., Maclaren, J., Herbst, M., Zaitsev, M., Turner, R., 2014. Prospective slice-by-slice motion correction reduces false positive activations in $\mathrm{fMRI}$ with task-correlated motion. Neuroimage 84, 124-132.

Scouten, A., Constable, R.T., 2007. Application and limitations of whole-brain MAGIC VASO fuctional fmaging. Magn Reson Med 58, 306-315.

Setsompop, K., Gagoski, B.A., Polimeni, J.R., Witzel, T., Wedeen, V.J., Wald, L.L., 2012a. Blippedcontrolled aliasing in parallel imaging for simultaneous multislice echo planar imaging with reduced g-factor penalty. Magn Reson Med 67, 1210-1224.

Setsompop, K., Gagoski, B.A., Polimeni, J.R., Witzel, T., Wedeen, V.J., Wald, L.L., 2012b. Blippedcontrolled aliasing in parallel imaging for simultaneous multislice echo planar imaging with reduced g-factor penalty. Magn Reson Med 67, 1210-1224. 
Stelzer, J., Lohmann, G., Mueller, K., Buschmann, T., Turner, R., 2014. Deficient approaches to human neuroimaging. Front Hum Neurosci 8, 462.

Trampel, R., Schäfer, A., Huber, L., Heidemann, R.M., Turner, R., 2013. Negative BOLD in somatosensory cortex during simple finger tapping. Proceedings of the Inernational Society of Magnetic Resonance in Medicine, p. 2339.

Turner, R., 2002. How much cortex can a vein drain? Downstrean milustion of activation-related cerebral blood oxygenation changes. Neuroimage 16, 1062-1067.

Uludağ, K., Muller-Bierl, B., Ugurbil, K., 2009. An integrative model for neuronal activity- induced signal changes for gradient and spin echo functional imaging. Neuroimage 48, 150-165.

van Zijl, P.C.M., Hua, J., Lu, H. 2012 The BOLD post-stimulus undershoot, one of the most debated issues in fMRI. Neurolmage 62, 1092-1102.

Wang, Y., Moeller, S., Li, X., Vu, A.T., Krasileva, K., Ugurbil, K., Yacoub, E., Wang, D.J., 2015. Simultaneous multi-slice Turbo-FLASH imaging with CAIPIRINHA for whole brain distortionfree pseudo-continuous arterial spin labeling at 3 and 7T. Neuroimage 113, 279-288.

Wolff S.D., Balaban R.S., Magnetization transfer contrast (MTC) and tissue water proton relaxation in vivo. Magn Reson Med 1989;10:135- 144.

Wong, E., 2012. Optimized phase schedules for minimizing peak RF power in simultaneous multi-slice RF excitation pulses. In Proceedings of the 20th Annual Meeting of ISMRM, Melbourne, Victoria, Australia, p. 2209.

Worsley, K.J., 2001. Statistical analysis of activation images. In P. Jezzard, P. M. Matthews, S. M. Smith (eds). Functional MRI: An introduction to Methods. Oxford University Press, Oxford, UK, pp. 251.

Yacoub, E., Van DeMoortele, P.F., Shmuel, A., Ugurbil, K., 2005. Signal and noise character- istics of Hahn SE and GE BOLD fMRI at $7 \mathrm{~T}$ in humans. Neuroimage 24, 738-750.

Yacoub, E., Harel, N., Ugurbil, K., 2008. High-field fMRI unveils orientation columns in humans. Proc. Natl. Acad. Sci. U. S. A. 105, 10607-10612.

Zhang, X., Petersen, E.T., Ghariq, E., De Vis, J.B., Webb, A.G., Teeuwisse, W.M., Hendrikse, J., van Osch, M.J., 2012. In vivo blood T(1) measurements at 1.5 T, 3 T, and 7 T. Magn Reson Med.

Zhao, F., Wang, P., Hendrich, K., Ugurbil, K., Kim, S.G., 2006. Cortical layer-dependent BOLD and CBV responses measured by spin-echo and gradient-echo fMRI: insights into hemodynamic regulation. Neuroimage 30, 1149-1160.

\section{Tables:}

Tab. 1: Statistical Z-value results of functional experiments.

\begin{tabular}{|c|c|c|c|c|c|c|}
\hline \multirow{2}{*}{ VASO } & \multicolumn{2}{|c|}{ Experiment $\mathrm{A}$} & \multicolumn{2}{|c|}{ Experiment B } & \multicolumn{2}{|c|}{ Experiment $\mathrm{C}$} \\
\hline & Mean & STD & Mean & STD & Mean & STD \\
\hline V1 area & 4.6 & 1.3 & \multicolumn{2}{|c|}{ ROI not in FOV } & 6.7 & 1.2 \\
\hline MT area & 3.7 & 1.2 & \multicolumn{2}{|c|}{ ROI not in FOV } & 4.6 & 1.1 \\
\hline $\begin{array}{c}\text { M1 area } \\
\text { (contralateral) }\end{array}$ & 3.7 & 0.5 & 5.5 & 2.2 & 8.0 & 3.2 \\
\hline
\end{tabular}

\begin{tabular}{|c|c|c|c|c|c|c|}
\hline \multirow{2}{*}{ BOLD } & \multicolumn{2}{|c|}{ Experiment $\mathrm{A}$} & \multicolumn{2}{|c|}{ Experiment B } & \multicolumn{2}{|c|}{ Experiment $\mathrm{C}$} \\
\hline & Mean & STD & Mean & STD & Mean & STD \\
\hline V1 area & 7.7 & 1.2 & \multicolumn{2}{|c|}{ ROI not in FOV } & 9.5 & 1.3 \\
\hline MT area & 4.5 & 1.8 & \multicolumn{2}{|c|}{ ROI not in FOV } & 6.3 & 0.7 \\
\hline $\begin{array}{c}\text { M1 area } \\
\text { (contralateral) }\end{array}$ & 7.2 & 1.4 & 9.7 & 2.3 & 10.5 & 3.1 \\
\hline
\end{tabular}


Figure captions:

Fig. 1: Magnetization preparation, readout, and sequence timing.

Schematic depiction of one TR in the SMS-VASO sequence starting with the application of an adiabatic inversion pulse. A phase skip is used to be in control of the inversion efficiency and inflowing fast blood. The VASO images are acquired around the blood nulling time at TI $1=$ $1.1 \mathrm{~s}$ after sequential transmission of multi-band RF excitation pulses. The multi-band factor varies between 2 and 4 in this study (SMS-factor $=4$ in figure). $\Delta T I$ denotes the deviation of the theoretical blood nulling time. Dependent on the acquisition parameters it is $\Delta \mathrm{TI}=75$ $175 \mathrm{~ms}$ in this study. The phase-encoding and read gradients for the 2D-EPI acquisition are accompanied with blipped-CAIPI gradients in slice direction for controlled aliasing of near slices. In this study the corresponding FoV-shift factor was between 1 and $1 / 3$ (here, FoVshift $=1 / 3$ ). A second set of images is acquired at $T 12=2.6 \mathrm{~s}$ containing BOLD signal weighting without $\mathrm{CBV}$-weighting.

Fig. 2: Results from experiment A: two slice groups during visuo-motor task.

SMS-VASO results for high-resolution imaging of the visuo-motor network containing V1, V5, M1, and S1. The left side of the figure refers to VASO-CBV sensitivity and the right side refers to the interleaved acquired BOLD signal. A depicts the imaging slab orientation, tSNR maps and the functional response of one representative subject. It can be seen, how the insensitivity of VASO to large draining veins results in an improved specificity to GM tissue compared to BOLD. The purple insets show how the activity clusters of VASO are confined to the two GM banks of the central sulcus, while BOLD signal shows one connected blob only. Also in V1, VASO activity patterns can delineate the cortex at the subarachnoid boundary better compared to GE-BOLD (blue arrow). B depicts the stability of the results across four participants. The higher specificity of VASO to GM tissue of M1 compared to GM tissue of S1 is visible consistently across participants. $\mathbf{C}$ depicts the corresponding time courses of BOLD and VASO signal in ROIs of V1, M1, and S1. Note that VASO is a negative contrast and VASO signal decrease is indicating CBV increase. Error bars refer to inter-participant standard deviations.

\section{Fig. 3: Results from experiment B: High-resolution imaging of the entire M1/S1 region.}

SMS-VASO results for high resolution imaging of the sensory-motor cortex. The left side of the figure refers to VASO-CBV sensitivity and the right side refers to the BOLD signal acquired interleaved. A depicts the imaging slab orientation, tSNR maps and the functional response of one representative subject. It can be seen how the insensitivity of VASO to large draining veins results in an improved specificity to GM tissue compared to BOLD. Activity clusters of VASO are confined to the two GM banks of the central sulcus, while BOLD signal shows one connected blob only. Note that the high specificity of VASO to GM tissue independent of large draining veins clearly reveals that M1 has a higher cortical thickness 
compared S1, which can be useful in cortical segmentation. B depicts the stability of the results across four participants. The higher specificity of VASO to GM tissue of M1 compared to $\mathrm{GM}$ tissue of $\mathrm{S} 1$ is visible consistently across participants. Please note that unlike Fig. 2, 4 this Fig. does not contain visual areas. The color code is chosen, such that red and blue stand for activation and deactivation during unilateral finger motion.

\section{6}

7

\section{Fig. 4: Results from experiment C: Near whole-brain coverage.}

SMS-VASO results for low-resolution near whole-brain coverage. The left side of the figure refers to VASO-CBV sensitivity and the right side refers to interleaved acquired BOLD signal. A depicts the imaging slab orientation, tSNR maps and the functional response of one representative subject $\mathbf{B}$ depicts the stability of the results across four participants. Both, SMS-VASO and BOLD can detect significant activity in V1, in V5 and the M1/S1 area across participants.

\section{Fig. 5: Direct comparison of SMS-VASO and non-SMS-VASO.}

tSNR results for all three experimental protocols $(A-C)$ in three participants. tSNR maps with and without SMS refer to the same in-plane acquisition scheme. There is no apparent loss of image quality when applying SMS imaging compared to conventional single-band imaging. With the application of blipped-CAIPI and the leakBlock unaliasing, the tSNR reduction is below $15 \%$ in all protocols and there is no visible signal leakage between the simultaneously acquired slices. 
partial inversion

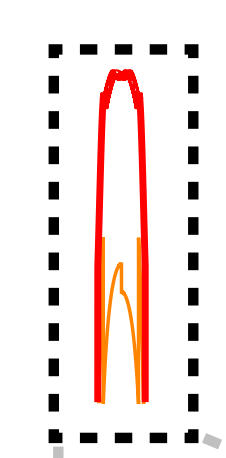

VASO excitation and readout

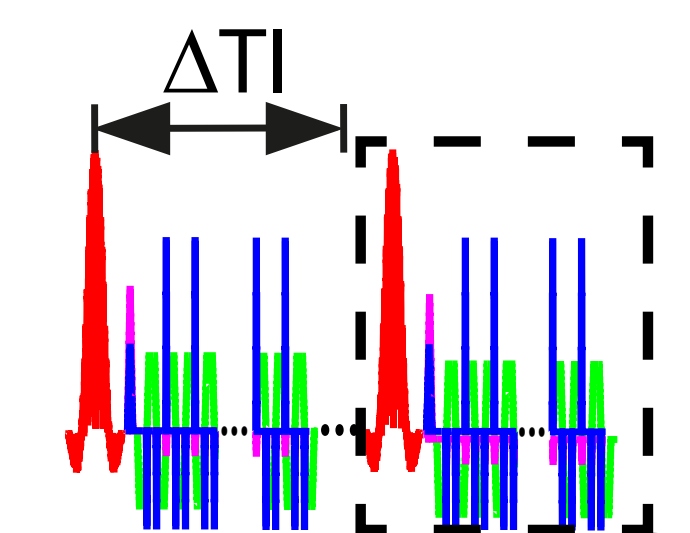

BOLD excitation and readout

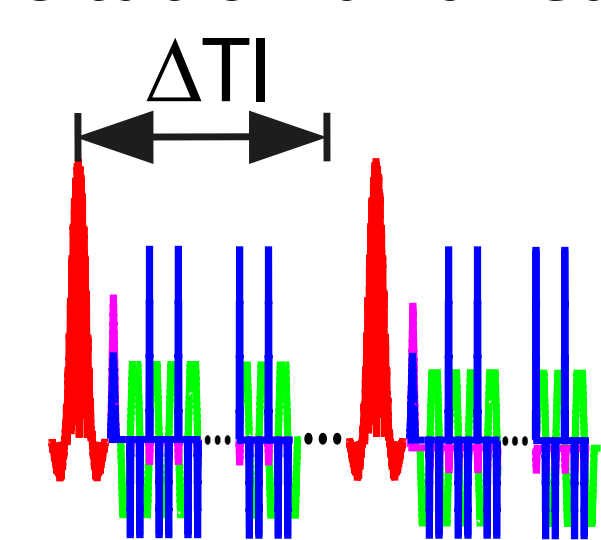

blood nulling time at TI1 $=1.1 \mathrm{~s}$

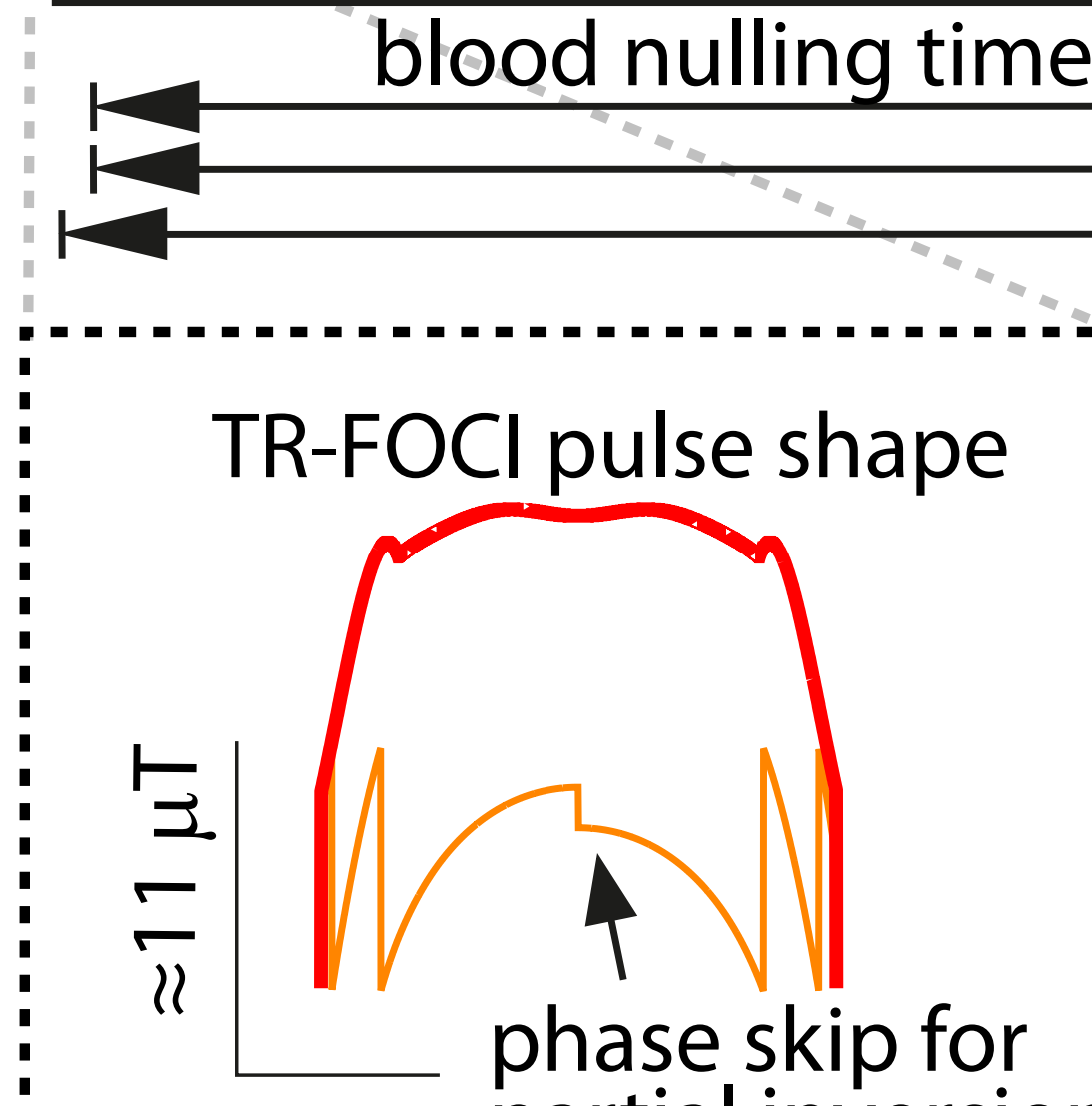

phase skip for 2 ms partial inversion:

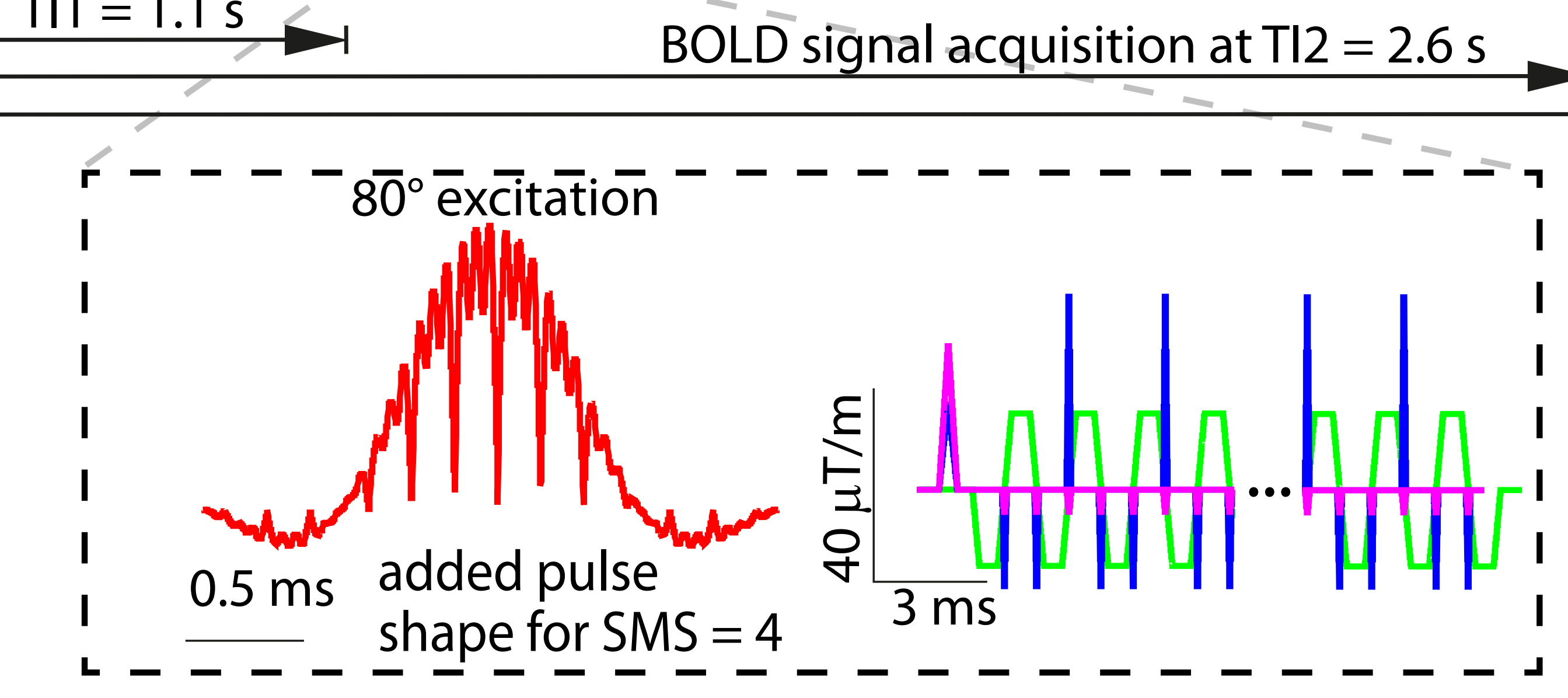

$\mathrm{TR}=3 \mathrm{~s}$

— RF amplitude

- RF phase

read gradient

- during EPI

- phase blips during EPI

- CAIPI blips 
A representative participant

VASO

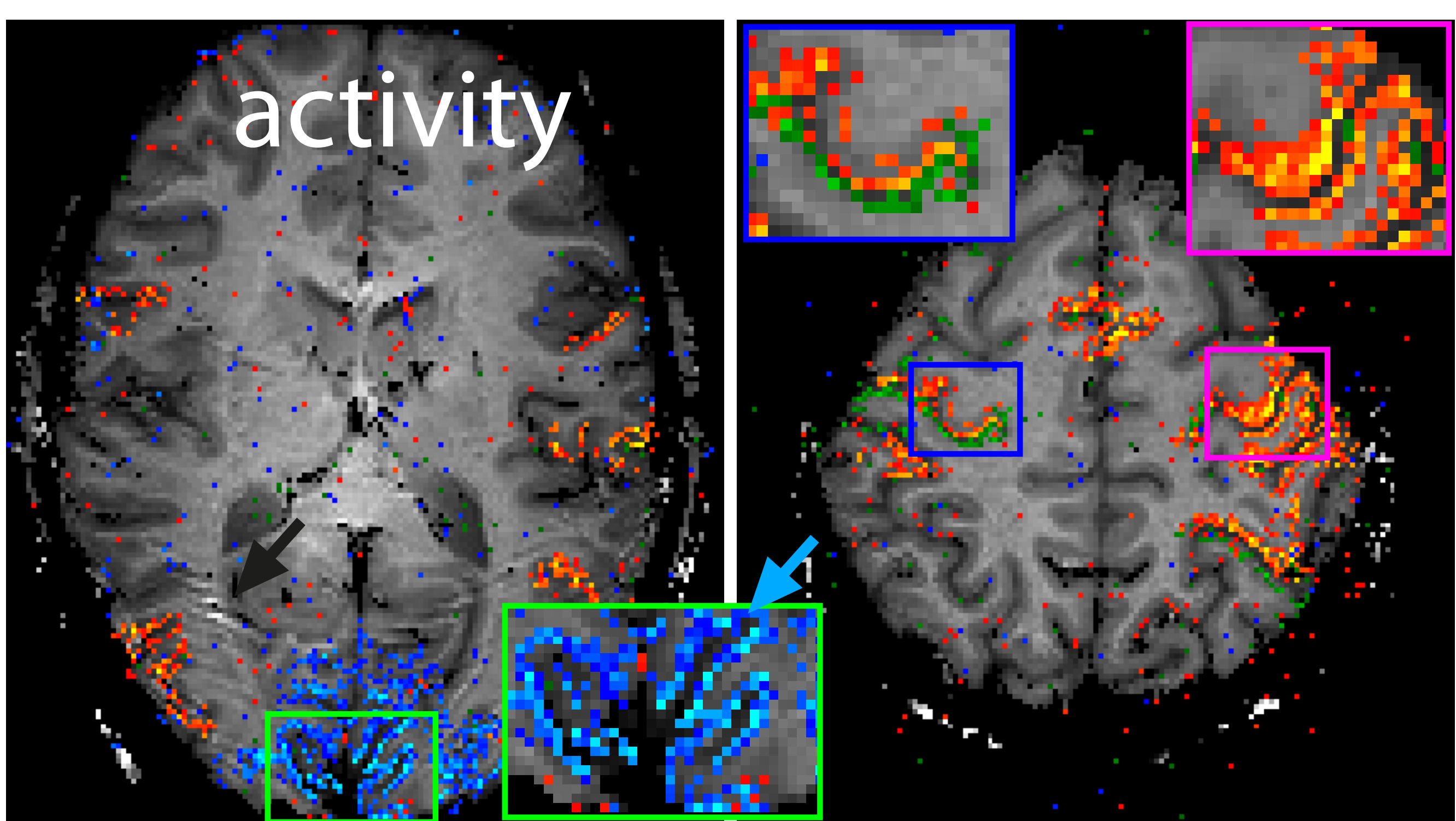

slice positions

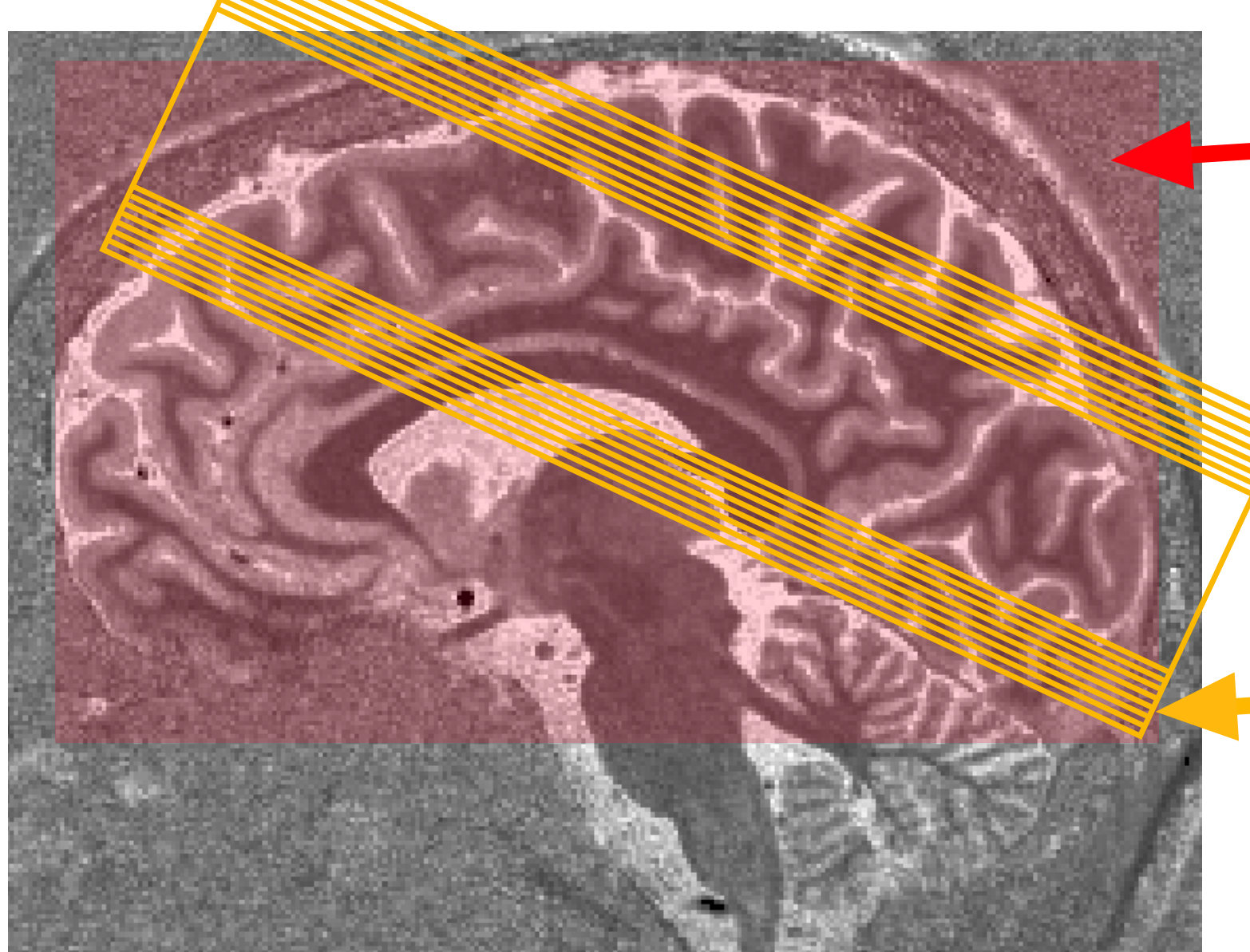

Z-val

BOLD 3 static 123 motion 203 static 12 VASO 1.7 rest 71.7 static 81.7 motion 8

slices

BOLD
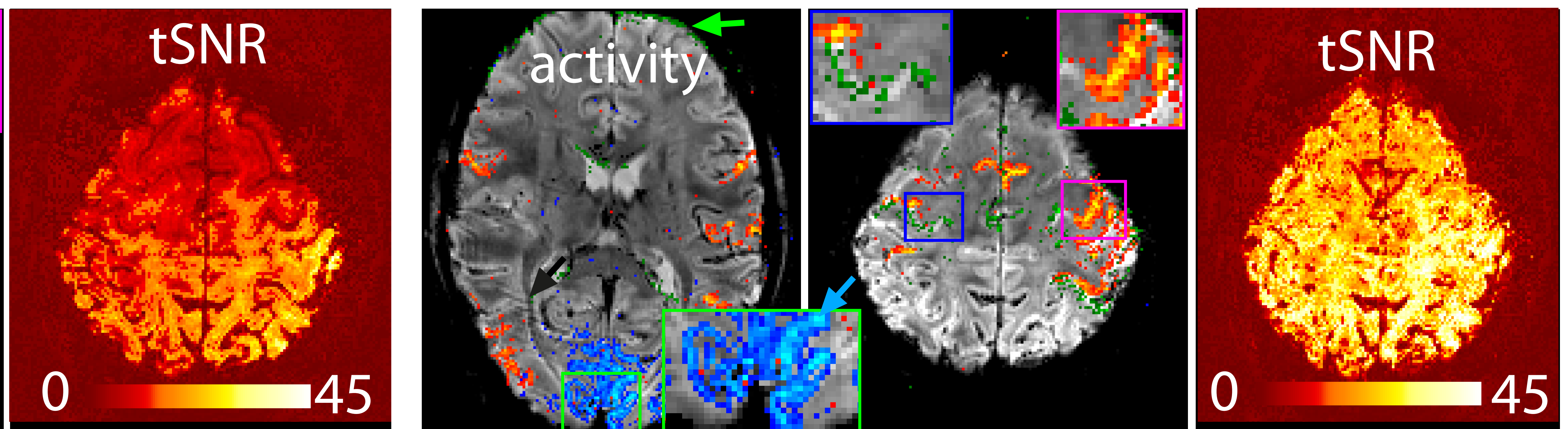

B consistency across participants
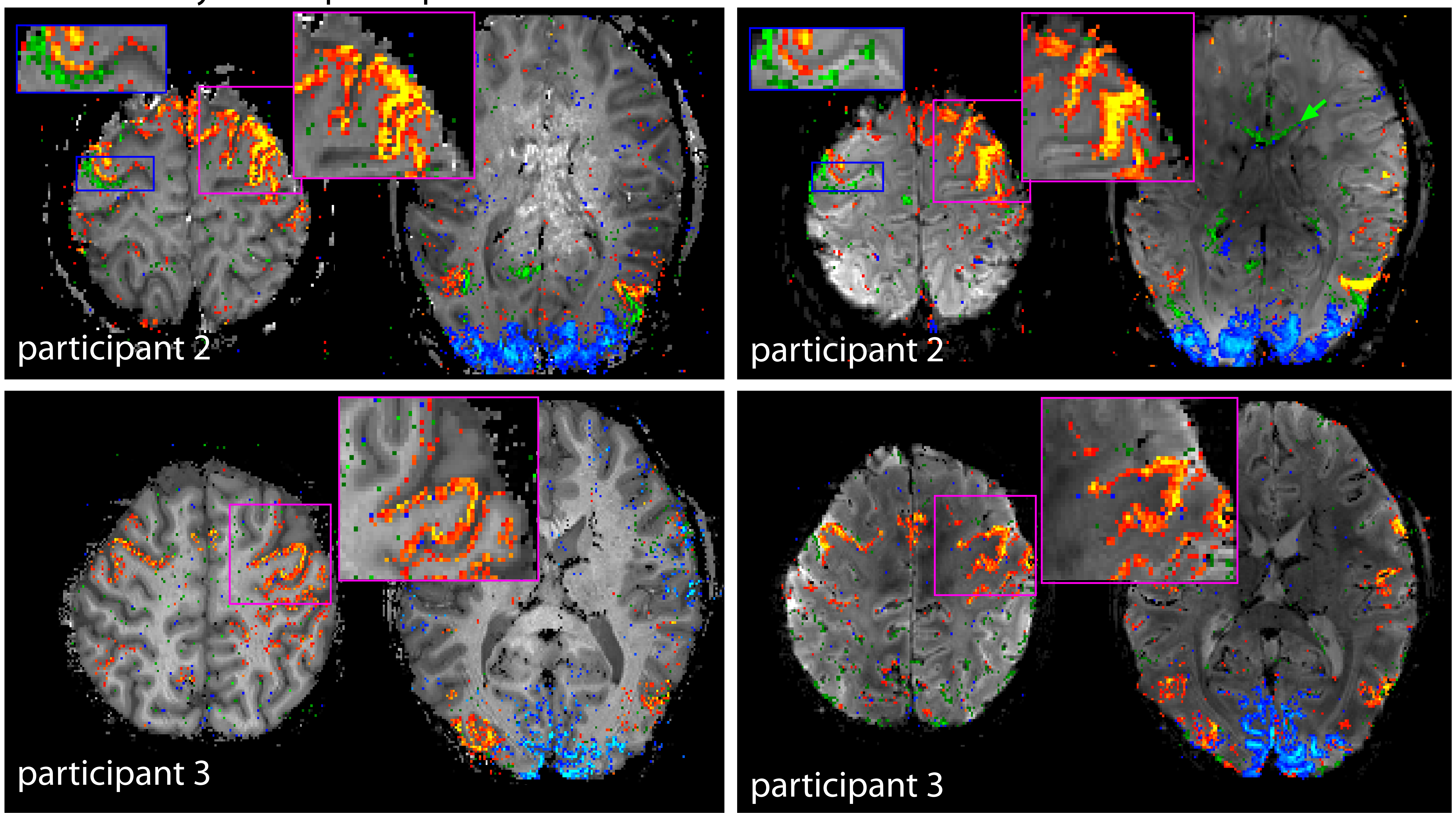

C time courses

$\frac{0}{2}$
$\frac{1}{0}$
$\frac{0}{n}$
$\frac{0}{n}$
$\frac{0}{0}$
$\frac{N}{\overline{0}}$
है
$\frac{0}{c}$

\%-BOLD V1

-BOLD V5

$0 \%$

-VASO V5

time $[s] \quad 0 \quad 10 \quad 20 \quad 30 \quad 40 \quad 50 \quad 60$

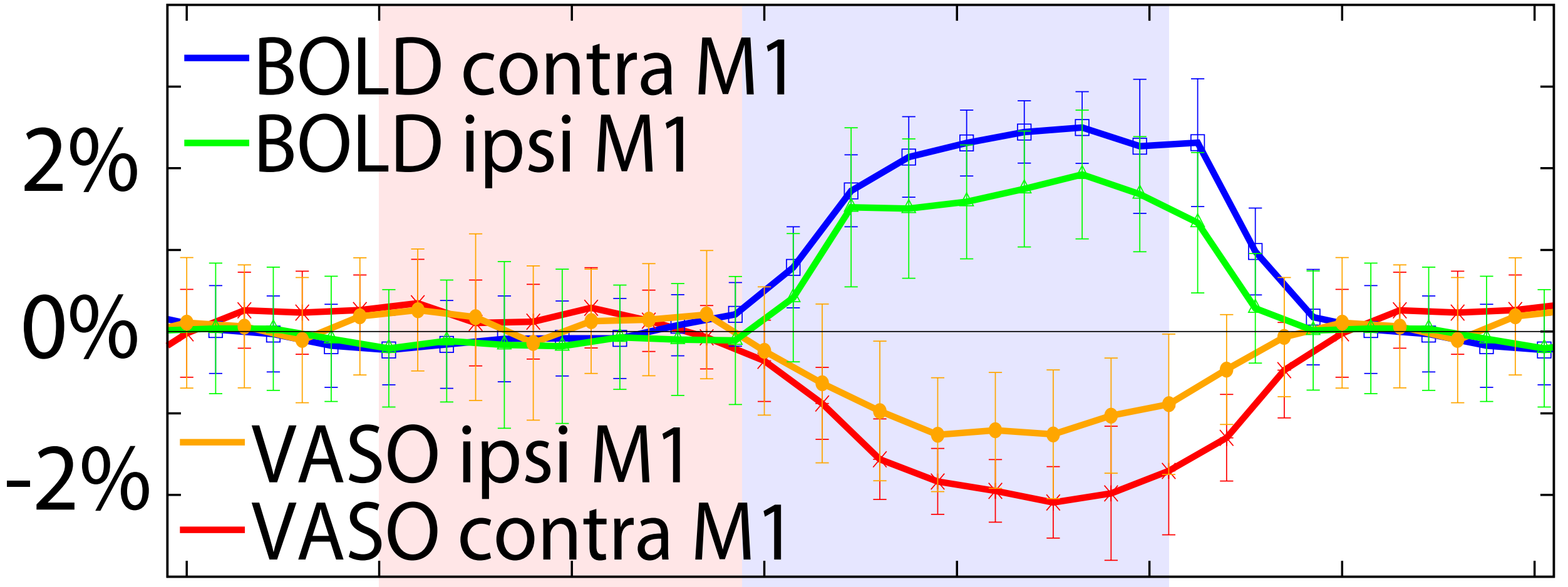

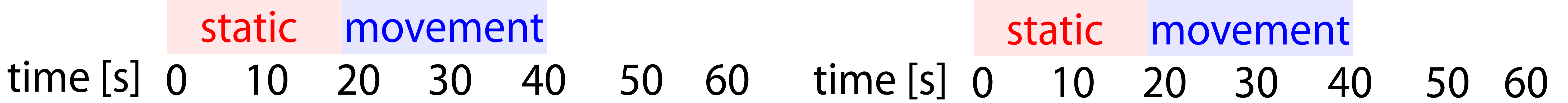

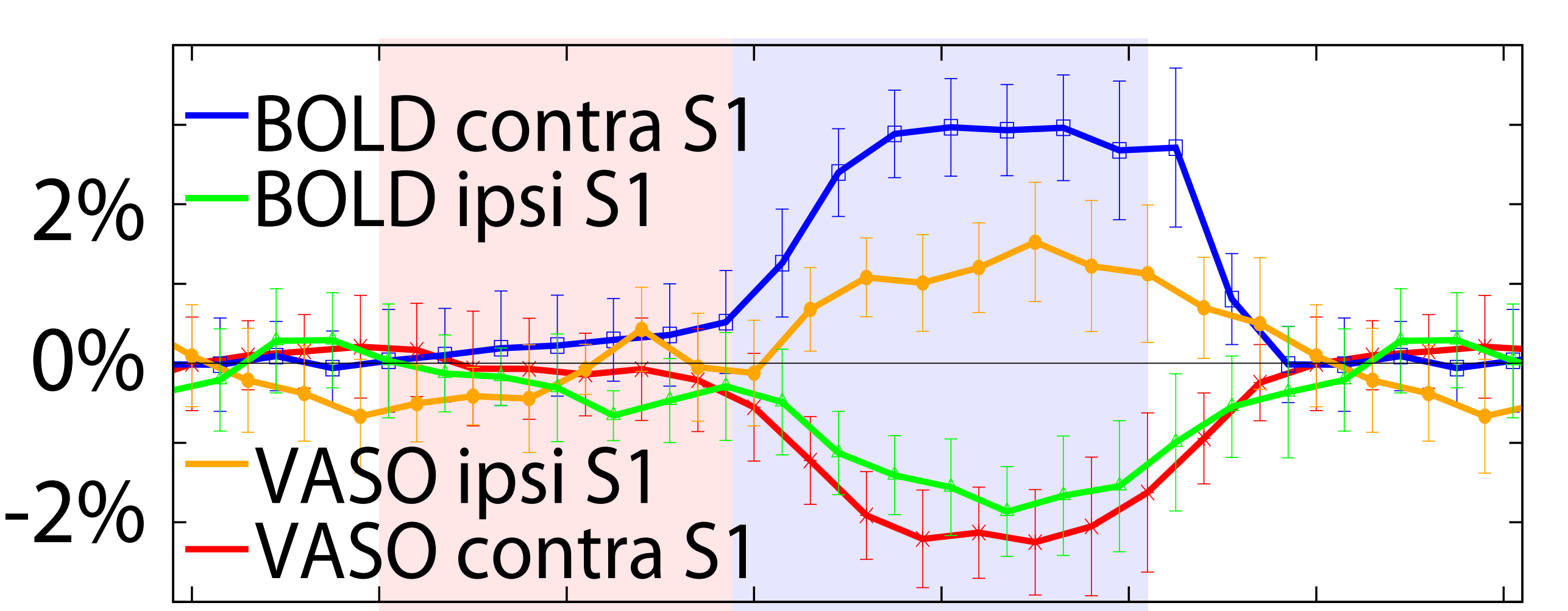

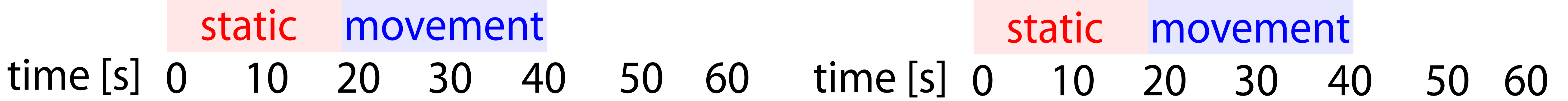




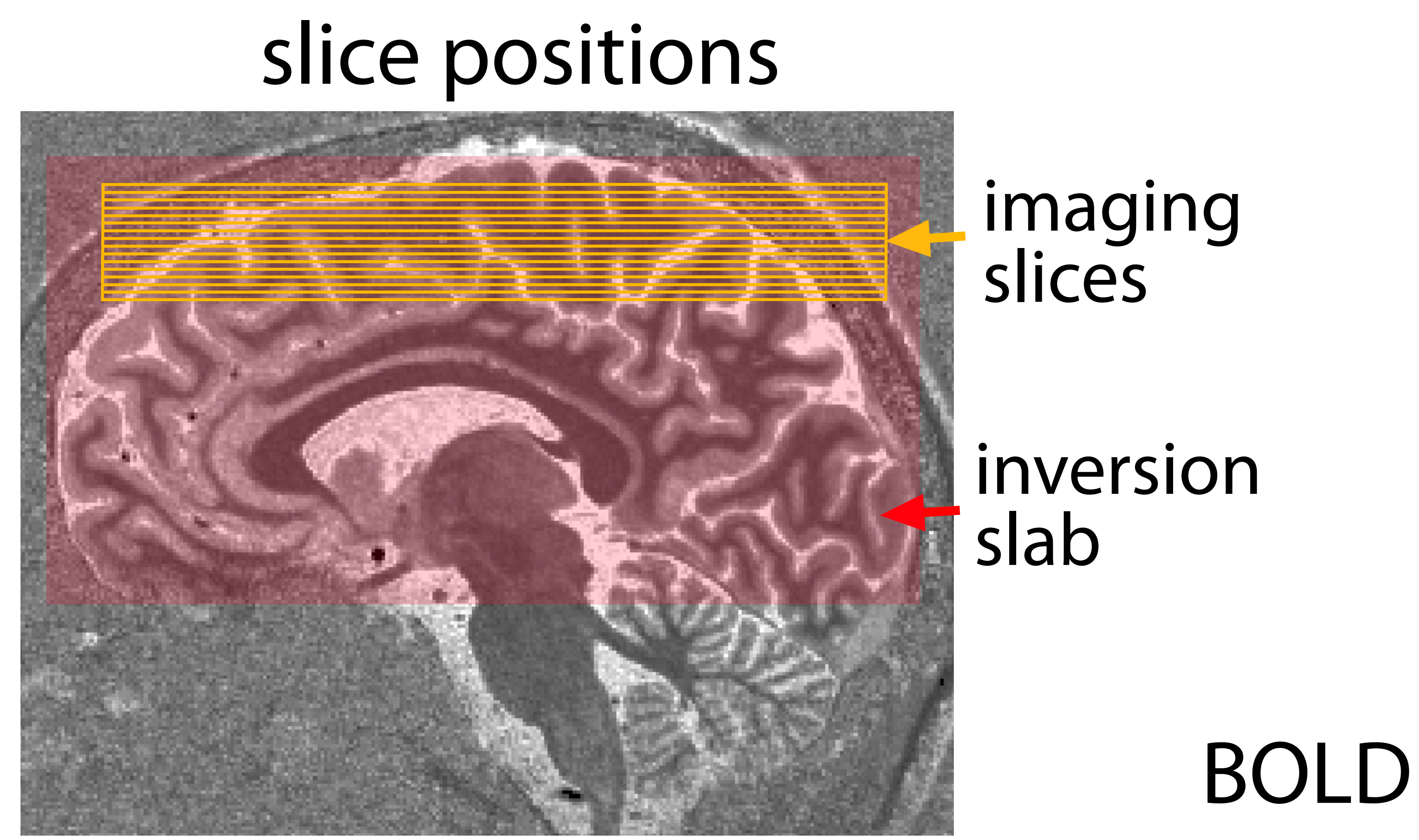

\begin{tabular}{lll|ccl|l} 
Z-val & 4 & tapping & 21 & 3 & rest vs. & 8 \\
BOLD & 2 & vs. rest & 9 & 2 & tapping & 5 \\
VASO & & V.
\end{tabular}

VASO
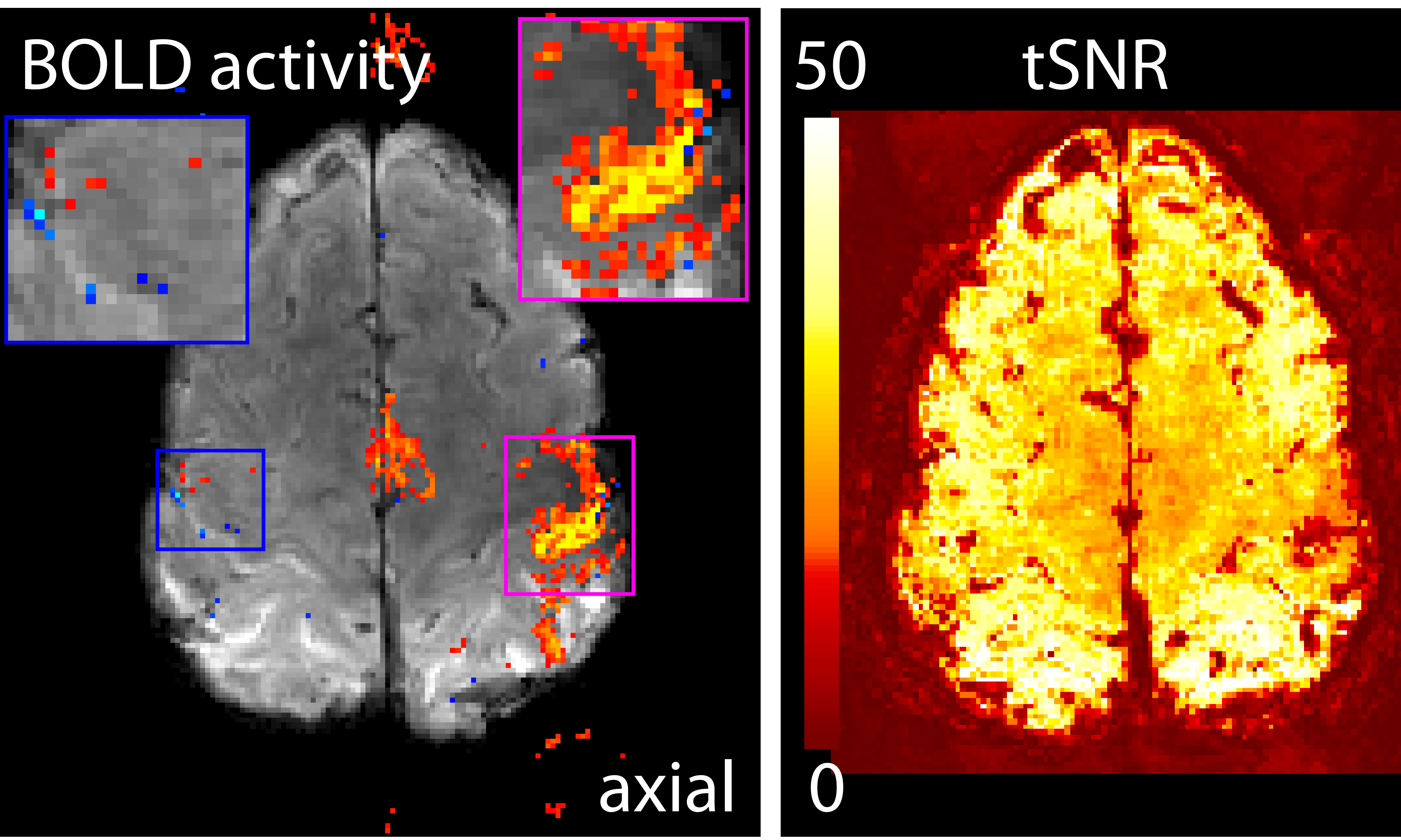
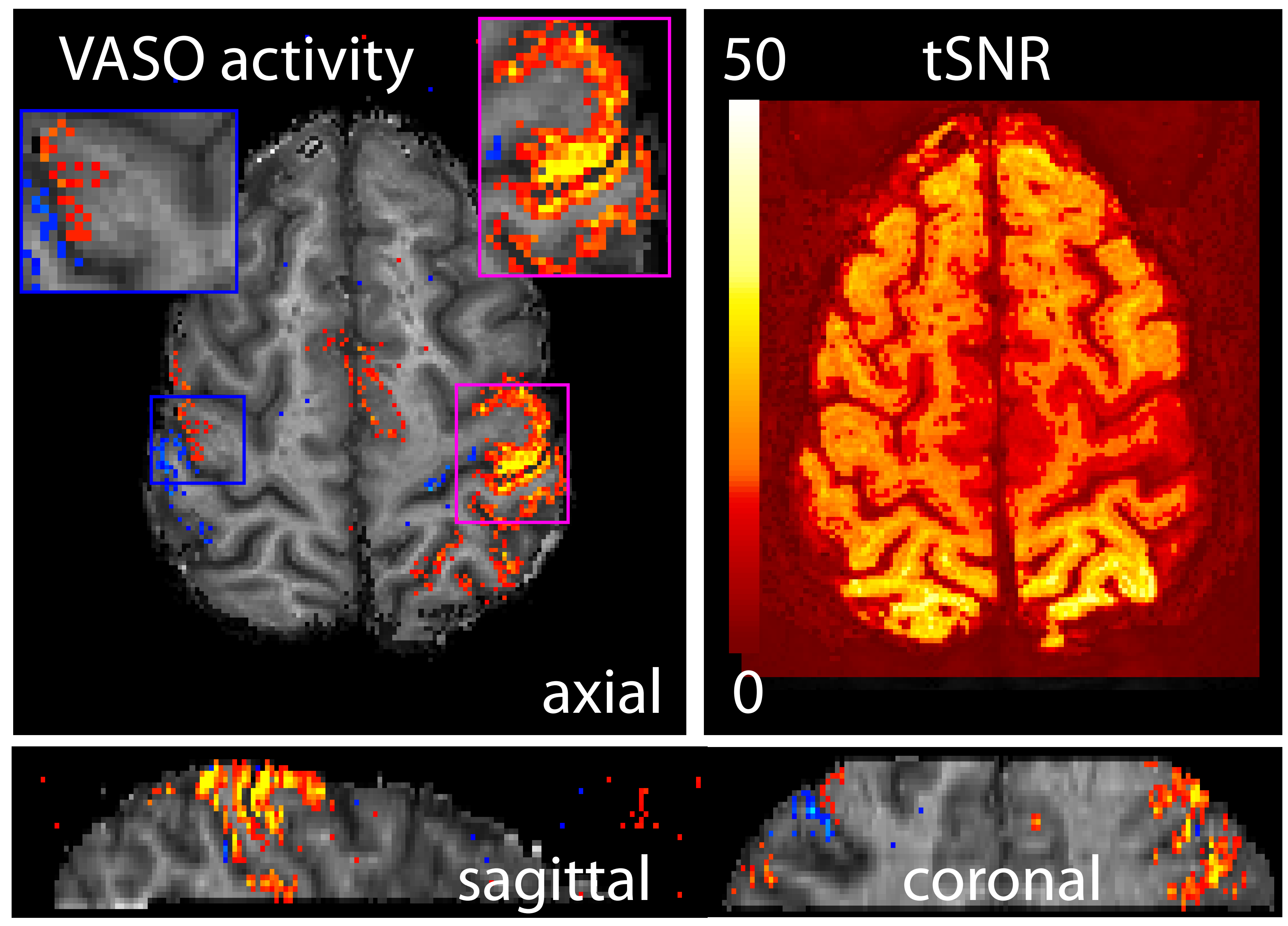

sagittal

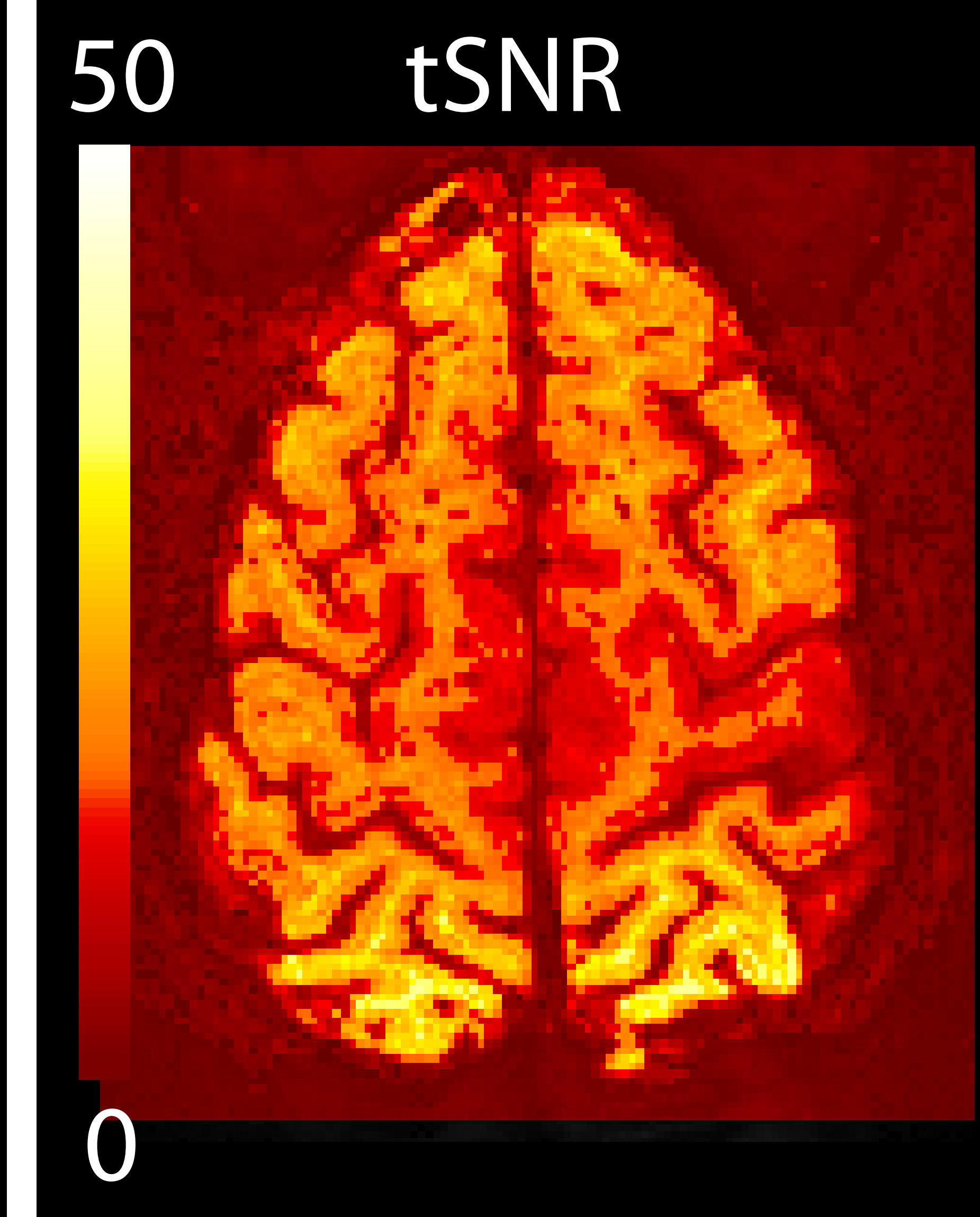

B consistency across participants
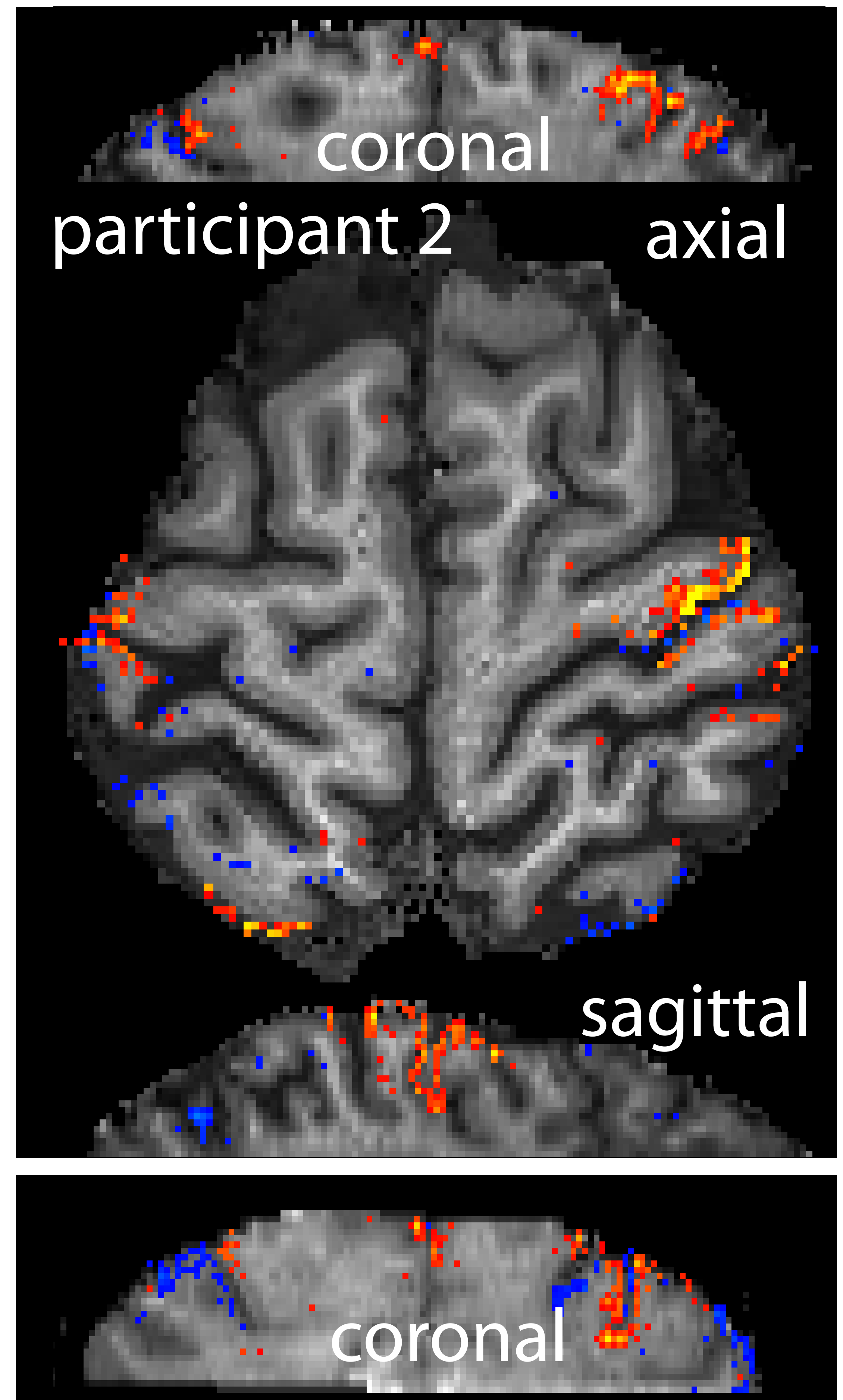

participant 4

axial

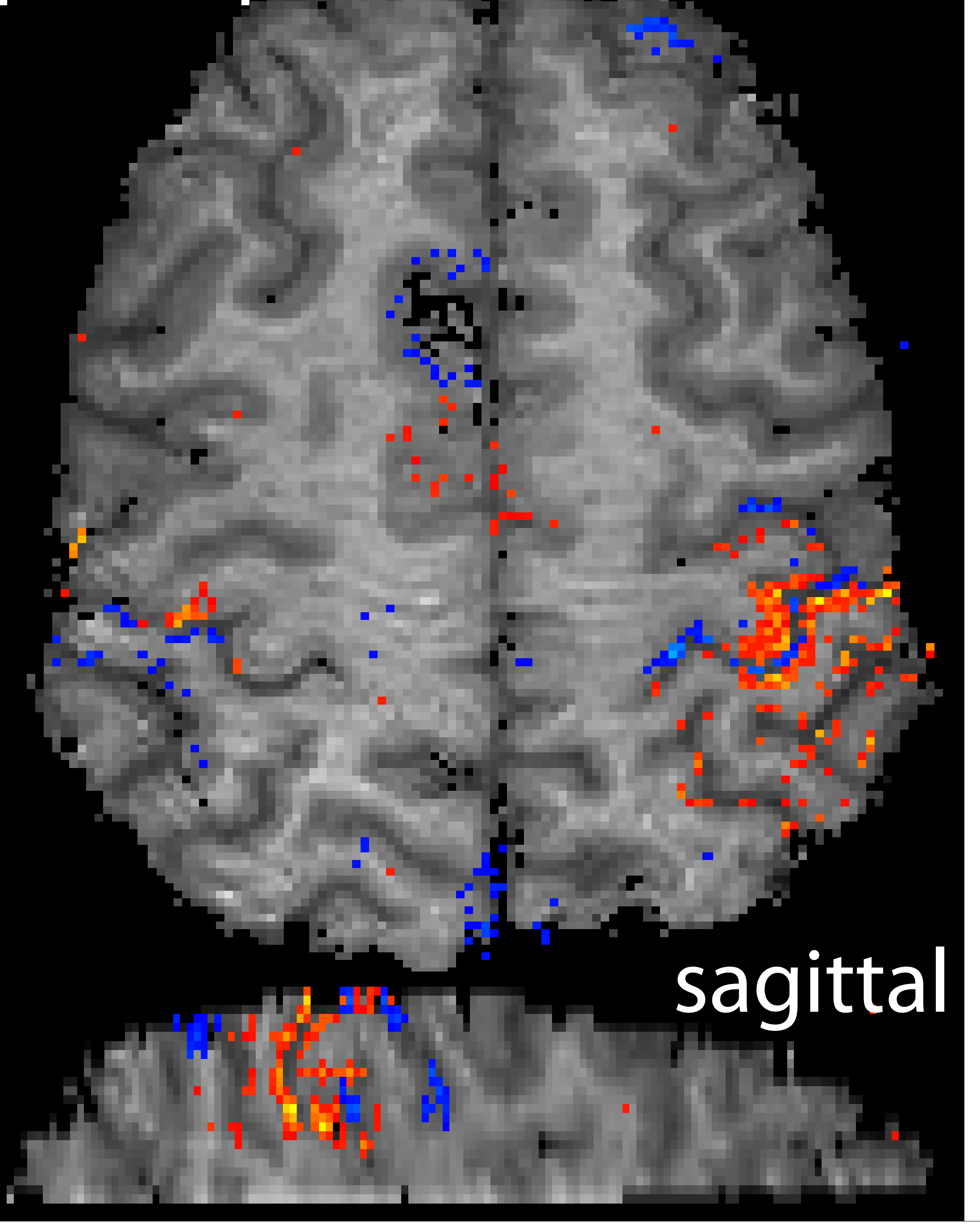

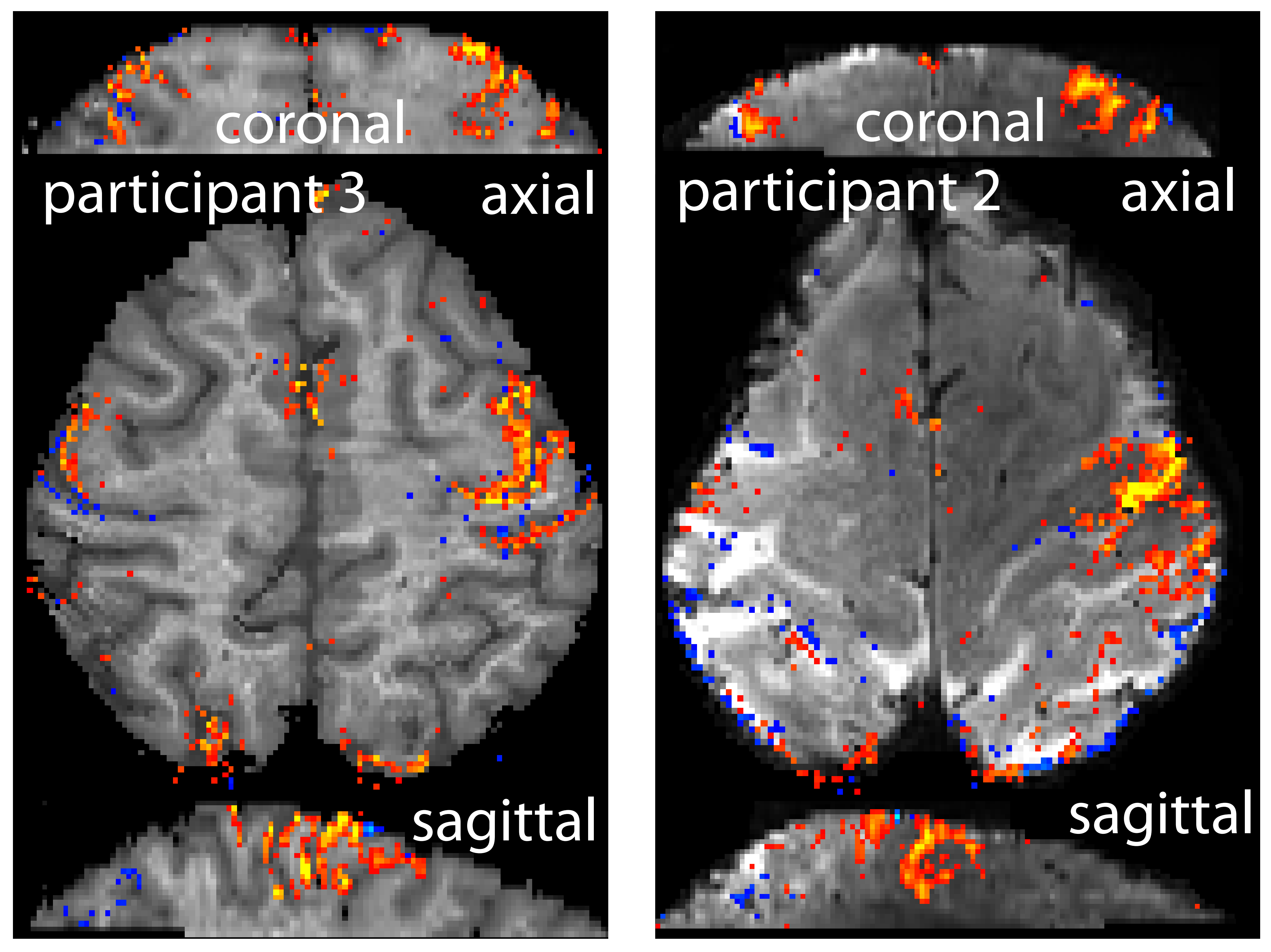
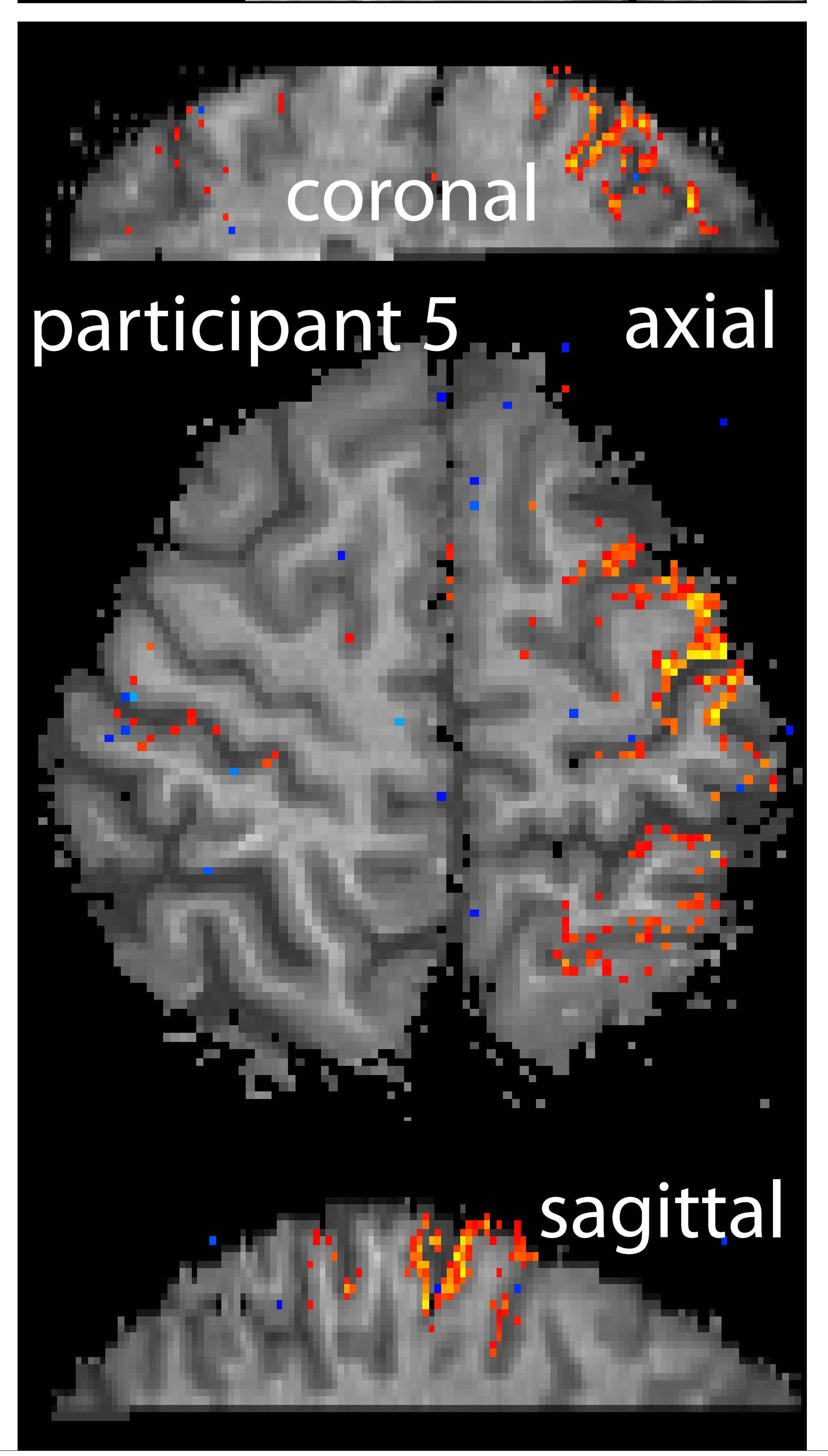
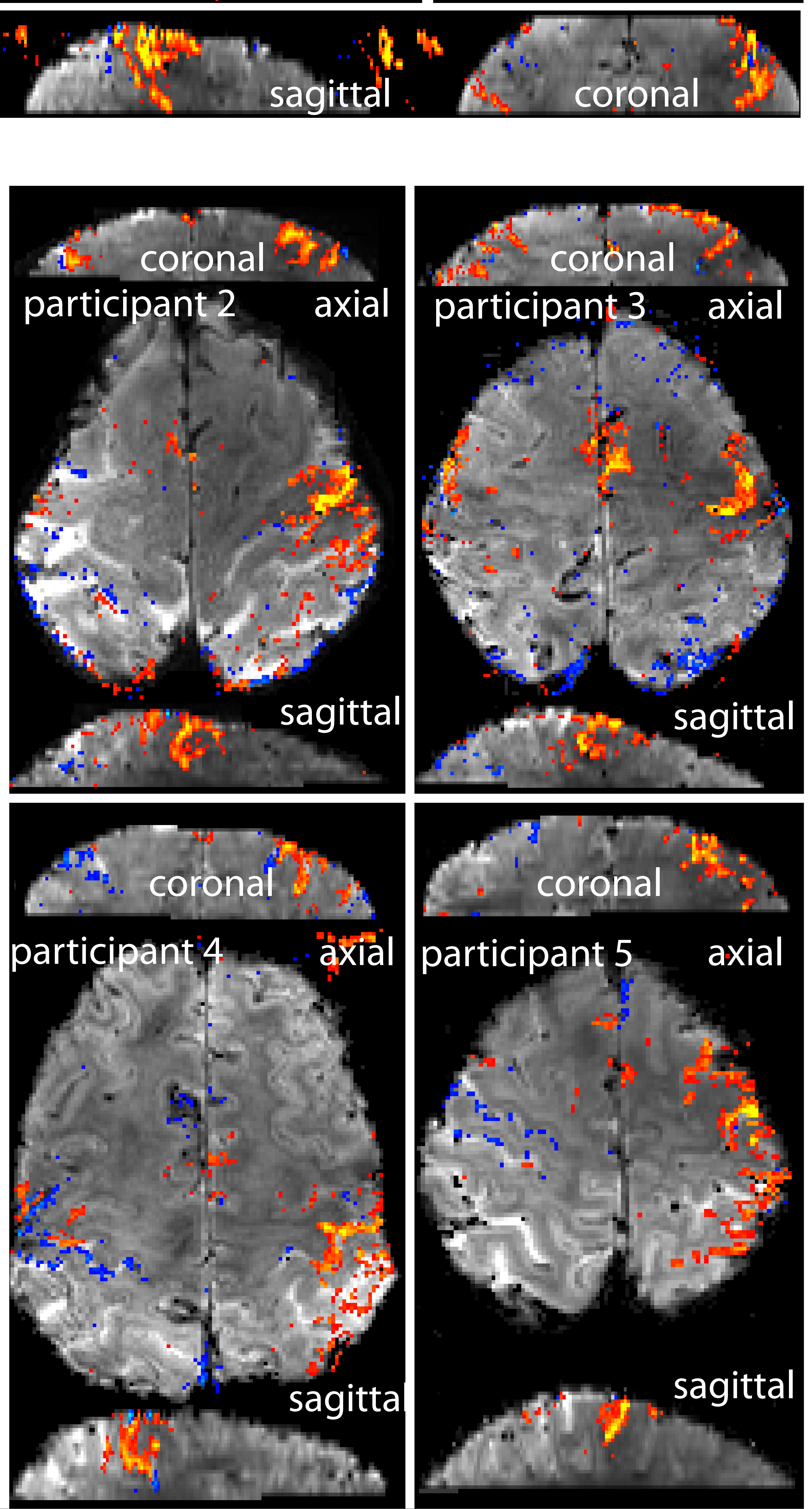

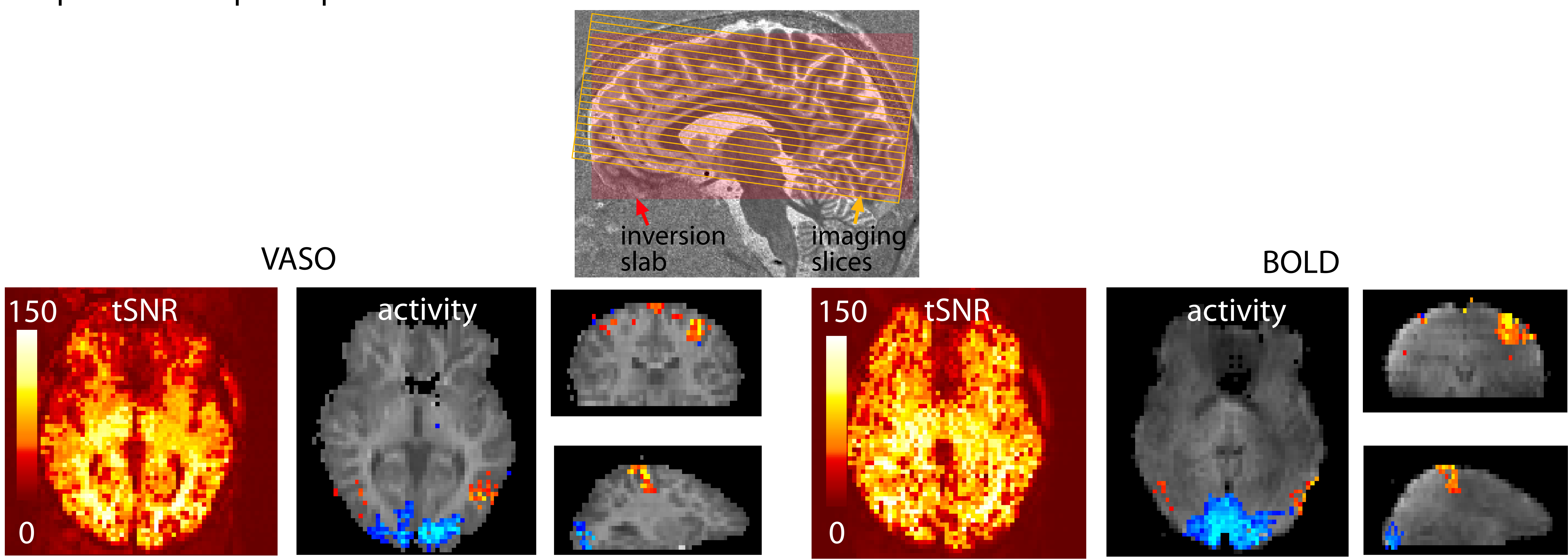

\section{B consistency across participients}
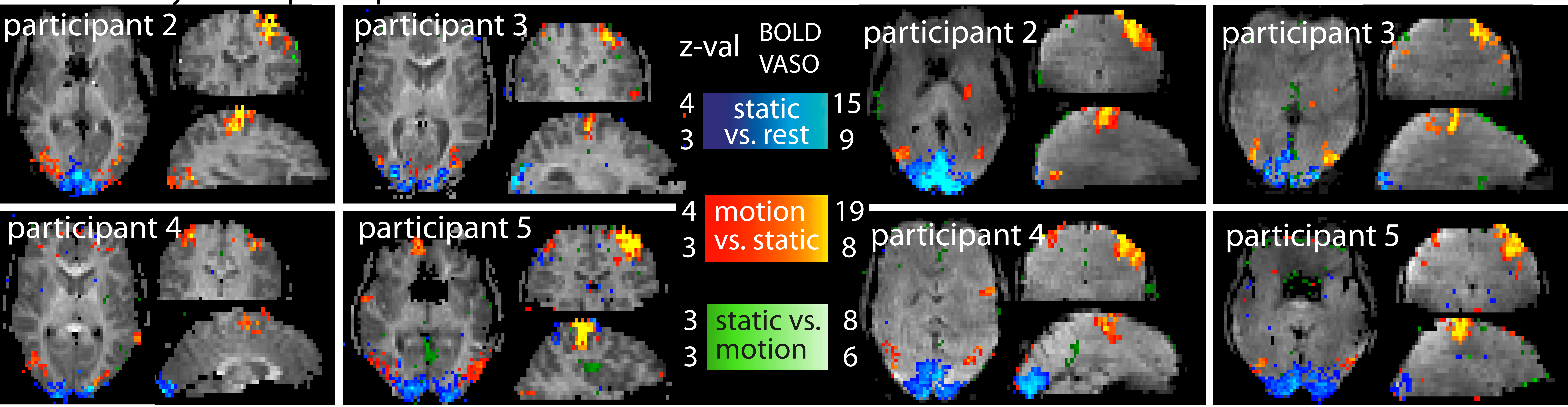


\section{SMS non-SMS SMS non-SMS SMS non-SMS}

2 slice groups
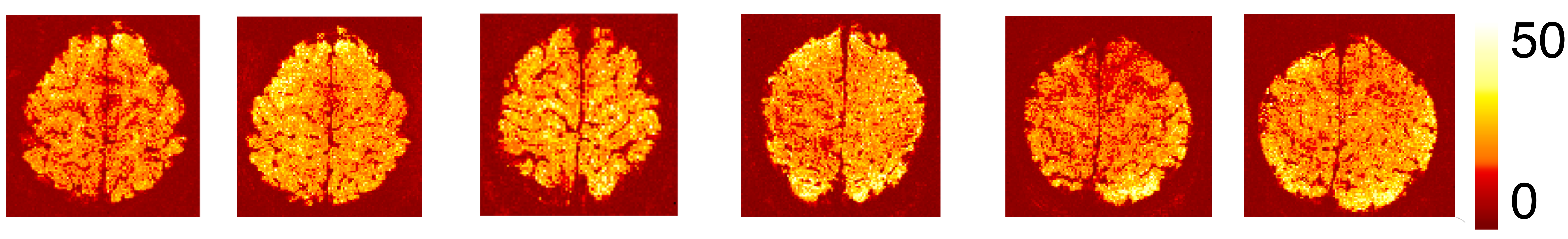

whole brain coverage
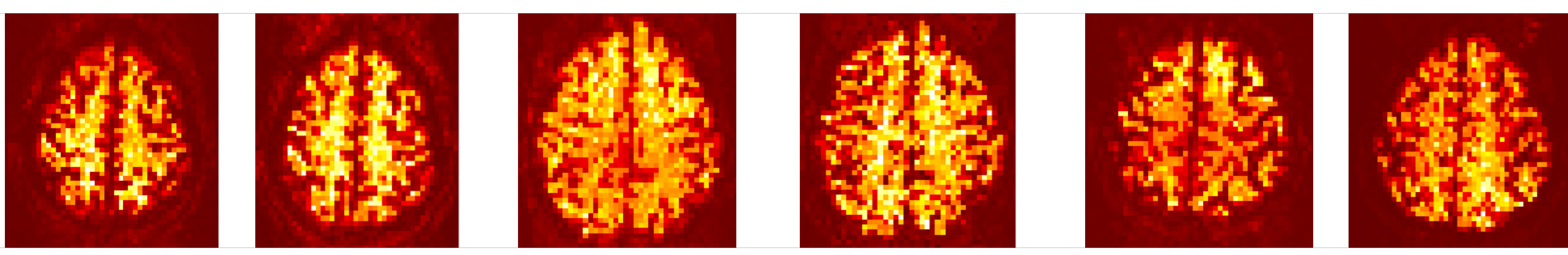

150

thick highres imaging slab
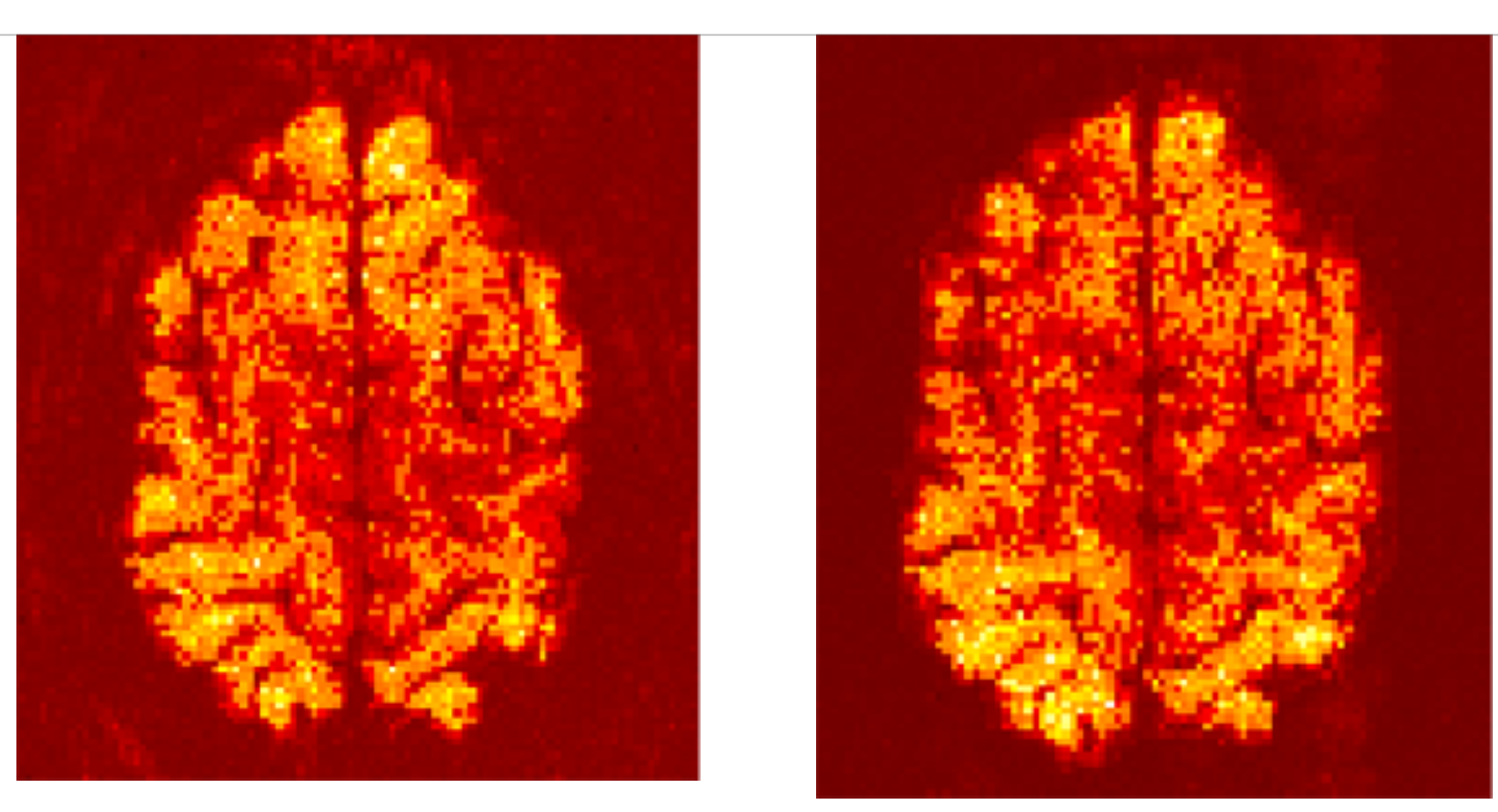

participant 1
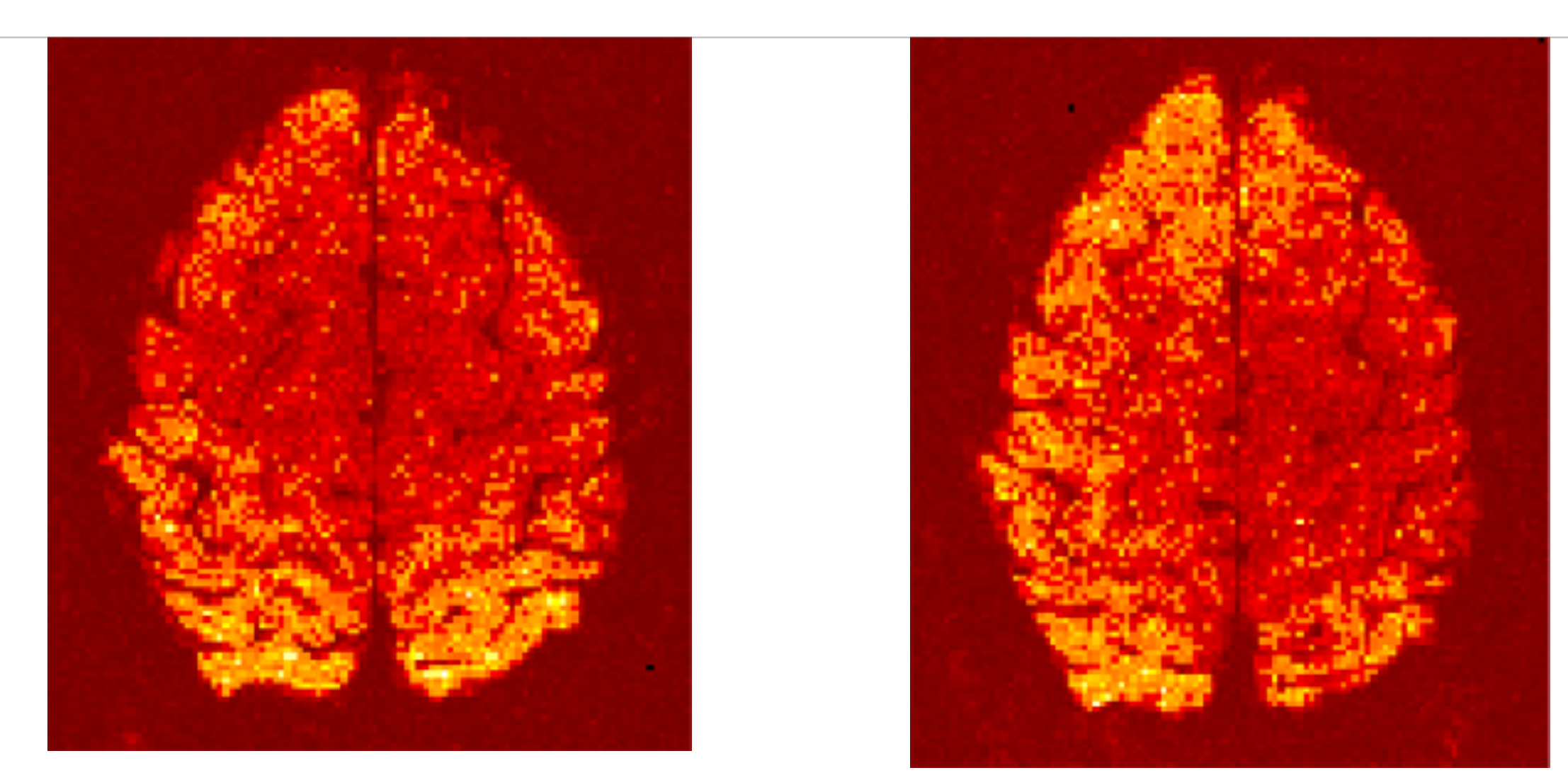

participant 2
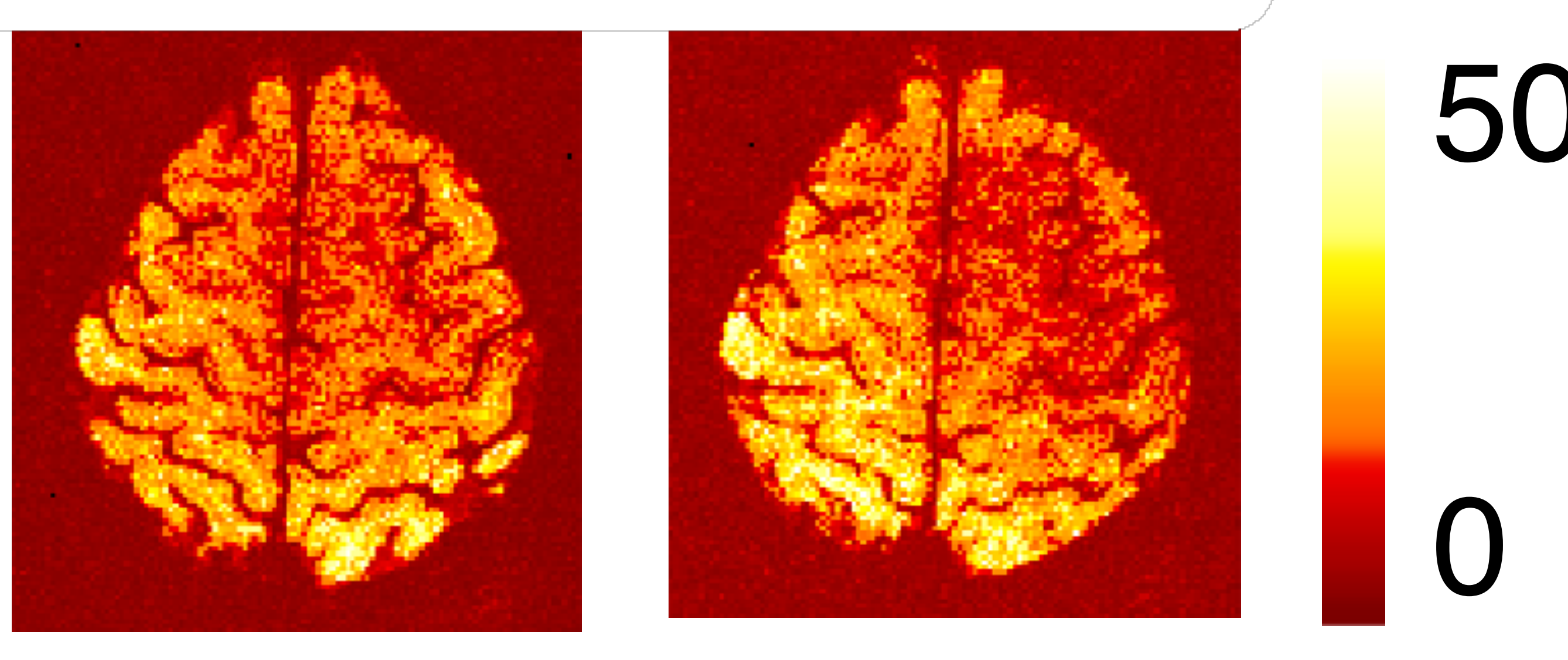

participant 3 